\title{
Dynamical Dark Matter from thermal freeze-out
}

\author{
Keith R. Dienes, ${ }^{1,2, *}$ Jacob Fennick, ${ }^{3, \dagger}$ Jason Kumar, ${ }^{4, \$}$ and Brooks Thomas ${ }^{5,8}$ \\ ${ }^{1}$ Department of Physics, University of Arizona, Tucson, Arizona 85721, USA \\ ${ }^{2}$ Department of Physics, University of Maryland, College Park, Maryland 20742, USA \\ ${ }^{3}$ Department of Mathematics, University of Hawaii, Honolulu, Hawaii 96822, USA \\ ${ }^{4}$ Department of Physics and Astronomy, University of Hawaii, Honolulu, Hawaii 96822, USA \\ ${ }^{5}$ Department of Physics, Lafayette College, Easton, Pennsylvania 18042, USA
}

(Received 11 January 2018; published 20 March 2018)

\begin{abstract}
In the Dynamical Dark-Matter (DDM) framework, the dark sector comprises a large number of constituent dark particles whose individual masses, lifetimes, and cosmological abundances obey specific scaling relations with respect to each other. In particular, the most natural versions of this framework tend to require a spectrum of cosmological abundances which scale inversely with mass, so that dark-sector states with larger masses have smaller abundances. Thus far, DDM model-building has primarily relied on nonthermal mechanisms for abundance generation such as misalignment production, since these mechanisms give rise to abundances that have this property. By contrast, the simplest versions of thermal freeze-out tend to produce abundances that increase, rather than decrease, with the mass of the dark-matter component. In this paper, we demonstrate that there exist relatively simple modifications of the traditional thermal freeze-out mechanism which "flip" the resulting abundance spectrum, producing abundances that scale inversely with mass. Moreover, we demonstrate that a far broader variety of scaling relations between lifetimes, abundances, and masses can emerge through thermal freeze-out than through the nonthermal mechanisms previously considered for DDM ensembles. The results of this paper thus extend the DDM framework into the thermal domain and essentially allow us to "design" our resulting DDM ensembles at will in order to realize a rich array of resulting dark-matter phenomenologies.
\end{abstract}

DOI: $10.1103 /$ PhysRevD.97.063522

\section{INTRODUCTION}

Dynamical Dark Matter (DDM) $[1,2]$ is a framework for dark-matter physics in which the dark sector is composed of a large ensemble of dark states exhibiting a variety of masses, lifetimes, and cosmological abundances. The phenomenological viability of this framework rests on a balancing between the lifetimes and abundances of the individual ensemble constituents, so that states with larger abundances have longer lifetimes while states with smaller abundances can have correspondingly smaller lifetimes. Such a balancing is required in order to satisfy observational constraints on dark-matter decay.

Scenarios within the DDM framework give rise to distinctive signatures at colliders [3,4], at direct-detection

\footnotetext{
*dienes@email.arizona.edu

jfennick@hawaii.edu

*jkumar@hawaii.edu

\$thomasbd@lafayette.edu
}

Published by the American Physical Society under the terms of the Creative Commons Attribution 4.0 International license. Further distribution of this work must maintain attribution to the author(s) and the published article's title, journal citation, and DOI. Funded by SCOAP ${ }^{3}$. experiments [5], and at indirect-detection experiments [6-8]. Such scenarios also give rise to enhanced complementarities $[9,10]$ between different types of experimental probes. Moreover, DDM ensembles have been shown to arise naturally in a number of scenarios for new physics beyond the Standard Model (SM). These include theories with extra spacetime dimensions $[1,2,11]$, theories involving strongly coupled hidden sectors [12], theories involving large spontaneously broken symmetry groups [13], and even string theories $[12,14]$. In these and other realistic DDM scenarios, the masses, lifetimes, and abundances of these individual particles are not arbitrary. Rather, these quantities follow directly from the underlying physics model and generally take the form of scaling relations which dictate how these quantities scale relative to one another across the ensemble as a whole. Through these scaling relations, the properties of the ensemble constituents - and thus the properties of the ensemble itself-are completely specified through only a small number of free parameters. Thus, even though the number of particles which contribute to the total dark-matter abundance is typically quite large, DDM scenarios of this sort are every bit as predictive as traditional dark-matter scenarios.

One of the most fundamental of these scaling relations is the one describing the relationship between the masses of 
the individual ensemble constituents and their cosmological abundances. This scaling relation in turn depends crucially on the mechanism through which the abundances for these constituents are generated. Thus, the properties of this scaling relation depend not only on the underlying particle-physics model, but also on the underlying cosmological history in which it is embedded. For example, in DDM models in which the ensemble constituents are the Kaluza-Klein (KK) modes of an axionlike particle propagating in the bulk of a theory with extra spacetime dimensions, misalignment production provides a natural abundance-generation mechanism for these constituents $[1,2,11]$. Likewise, in DDM models in which the ensemble constituents are composite states in the confining phase of a strongly coupled hidden sector, it turns out that residual gauge interactions at temperatures just below the confinement scale give rise to an appropriate spectrum of abundances which compensates for the exponential rise in the density of states [12]. However, these abundance-generation mechanisms are only compatible with particular classes of particle-physics models. It is therefore useful to explore alternative mechanisms for abundance generationmechanisms which might be applicable in a broader variety of DDM contexts.

Of course, one of the most widely discussed and widely exploited methods of abundance generation in the darkmatter literature is thermal freeze-out (for reviews, see, e.g., Refs. [15-18]). Indeed, thermal freeze-out provides a natural and versatile mechanism through which a neutral, weakly interacting massive particle (WIMP) species $\chi$ which is initially in thermal equilibrium can acquire a present-day abundance $\Omega_{\chi}$ on the order of the total present-day darkmatter abundance $\Omega_{\mathrm{CDM}} \approx 0.26$ [19]. Indeed, this mechanism not only underpins the so-called "WIMP miracle," but also generically yields $\Omega_{\chi} \sim \Omega_{\mathrm{CDM}}$ for a broader class of dark-matter particles which do not participate in SM weak interactions but which nevertheless have annihilation crosssections similar to that of a traditional WIMP [20]. The range of dark-matter masses $m_{\chi}$ for which the freeze-out is typically relevant is $\mathcal{O}(1 \mathrm{keV}) \lesssim m_{\chi} \lesssim \mathcal{O}(100 \mathrm{TeV})$. The lower limit to this range stems from the requirement that the dark-matter candidate be "cold"-i.e., nonrelativistic-during the freeze-out epoch (see, e.g., Ref. [21]), while the upper limit stems from considerations related to perturbative unitarity [22]. However, there are ways of circumventing this upper bound and broadening the window of applicability. For example, this bound is considerably relaxed in theories in which the dark and hidden sectors thermally decouple well before the freeze-out epoch [23].

The question then arises as to whether thermal freeze-out can yield a spectrum of cosmological abundances that are suitable for a DDM ensemble. At first glance, it may seem that this is not possible. The reason is relatively simple. In a DDM ensemble, the abundances of the ensemble constituents must generally scale inversely to their decay widths.
However, the decay widths of such states generally scale as a positive power of the mass. This then requires the cosmological abundances of the ensemble constituents to scale inversely with their masses:

$$
\Omega_{i} \sim m_{i}^{\gamma} \text { where } \gamma<0 .
$$

Unfortunately, while this holds for all of the nonthermal production mechanisms that have thus far been exploited for DDM abundance generation, this is generally not a property of thermal freeze-out. Indeed, as we know, the WIMP miracle itself rests upon the classic observation that [20,24-27]

$$
\Omega_{\chi} \sim \frac{m_{\chi}^{2}}{g_{\chi}^{4}},
$$

implying the canonical value $\gamma=+2$. Thus, all else being equal, dark-matter particles with larger masses can be expected to retain larger cosmological abundances after freeze-out than those with smaller masses-precisely the opposite of what is generically needed for a DDM ensemble.

In this paper, we shall demonstrate that an acceptable spectrum of abundances can nevertheless be generated for a DDM ensemble through thermal freeze-out, with abundances $\Omega_{i}$ scaling inversely with masses $m_{i}$ across the ensemble. Indeed, this can occur even if the couplings $g_{i}$ are universal across all ensemble constituents. Moreover, as we shall demonstrate, such thermal freeze-out scenarios can arise completely naturally, without any fine-tuning. Indeed, we shall find that such scenarios can even give rise to a wide variety of possible scaling behaviors with a wide range of possible (negative) scaling exponents $\gamma$. Thus, from a model-building perspective, we shall find that thermal freeze-out actually provides a versatile tool for "designing" viable DDM ensembles with different scaling behaviors and exploring their resulting phenomenologies.

This paper is organized as follows. In Sec. II, we examine the ways in which the cosmological abundance of a particle produced by thermal freeze-out depends on the mass of that particle. We review how the canonical relationship between abundance and mass arises within the WIMP paradigm and illustrate how this relationship can be altered through modifications of the particle physics alone, without any modification of the background cosmology. In Sec. III, we then undertake a somewhat more general study along the lines of this approach and derive a general expression for the freeze-out cosmological abundance of an individual ensemble constituent as a function of the mass, spin, and couplings of the particles involved. In this way, we find that we can generate a broad range of negative scaling exponents $\gamma$ and potentially even dial between them. In Sec. IV, we then examine how and under what conditions a suitable balancing of decay widths against abundances-a balancing which is the hallmark of the DDM framework-can naturally arise in thermal 
(a)

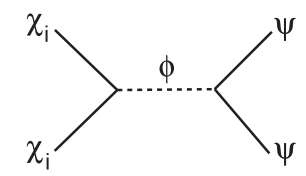

(b)

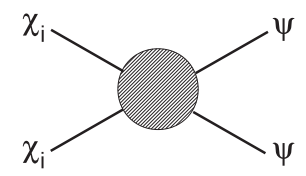

FIG. 1. Diagrams for dark-matter annihilation $\bar{\chi} \chi \rightarrow \bar{\psi} \psi$. (a) An $s$-channel diagram in which annihilation proceeds through a mediator $\phi$. (b) A four-point effective contact interaction which emerges from diagram (a) upon integrating out the mediator $\phi$. This diagram represents the limit of diagram (a) in which $m_{\phi} \gg m_{\chi}, m_{\psi}$.

DDM scenarios. Finally, in Sec. V, we conclude with a summary of the implications of our results for DDM model-building in a thermal context and possible directions for future work.

\section{FLIPPING THE ABUNDANCE SPECTRUM: INTEGRATING OUT PRIOR TO FREEZING OUT}

In general, thermal freeze-out of a given dark-matter particle $\chi$ results from a competition between $\chi$ annihilation and the Hubble expansion of the universe. A priori, we can imagine an annihilation process of the form $\bar{\chi} \chi \rightarrow \bar{\psi} \psi$ where $\psi$ denotes SM states or even states in the dark sector which are less massive than $\chi$. As the universe expands, the efficient annihilation and production of $\chi$ via this process and its reverse ensures that $\chi$ remains in thermal equilibrium with $\psi$ and all other particles that are in thermal equilibrium with $\psi$. Once the universe has cooled to a point at which $\chi$ is nonrelativistic, this thermal equilibrium causes $n_{\chi}$, the particle density of $\chi$, to fall exponentially as a function of time, which in turn causes the annihilation rate to fall as well. This situation persists until the annihilation rate $\Gamma$ falls below the Hubble parameter $H$. At this point, the expansion of the universe has caused $n_{\chi}$ to fall so low that the dark-matter particles can no longer efficiently find each other in order to annihilate. The efficient annihilation and production of $\chi$ then ends, with $\chi$ falling out of chemical equilibrium and the number of $\chi$ particles remaining essentially constant thereafter. In other words, $\chi$ has experienced thermal freeze-out.

Estimating the resulting post-freeze-out dark-matter abundance $\Omega_{\chi}$ therefore requires knowledge of the thermally averaged annihilation cross-section $\langle\sigma v\rangle$. Calculating this quantity in turn requires a set of specific assumptions concerning how $\chi$ annihilates into SM states or other relatively light states in the dark sector. In general, there are many processes which can contribute to the overall annihilation cross-section. However, for the purposes of this paper, we will concentrate on the relatively simple case in which this annihilation proceeds through an $s$-channel diagram such as that shown in Fig. 1(a) in which two darkmatter particles $\chi$ of mass $m_{\chi}$ annihilate into two light

particles $\psi$ through a mediator $\phi$ : i.e., $\bar{\chi} \chi \rightarrow \phi \rightarrow \bar{\psi} \psi$. Note that we are not assuming that $\chi$ or $\psi$ are their own antiparticles, nor are we even specifying the spins of these states. However, for simplicity, we shall begin by assuming that $m_{\chi} \gg m_{\phi}, m_{\psi}$ so that both $\phi$ and $\psi$ can be taken as effectively massless. We shall likewise take $g_{\chi}$ and $g_{\psi}$ to be constants representing the couplings of $\chi$ to $\phi$ and $\phi$ to $\psi$, respectively. We shall also take each of our incoming dark-matter particles to be nonrelativistic, with an energy $E_{\chi} \approx m_{\chi}$. It then immediately follows via dimensional analysis that our thermally averaged cross-section generically takes the form [20,24-27]

$$
\langle\sigma v\rangle \sim \frac{g_{\chi}^{2} g_{\psi}^{2}}{m_{\chi}^{2}}
$$

where $v$ denotes the relative velocity of the dark-matter particles. As we shall discuss below, under rather broad assumptions the process of thermal freeze-out leads to a residual abundance $\Omega_{\chi}$ which scales as $\langle\sigma v\rangle^{-1}$. This then reproduces the traditional "WIMP miracle" result

$$
\Omega_{\chi} \sim \frac{m_{\chi}^{2}}{g_{\chi}^{2} g_{\psi}^{2}},
$$

thereby yielding the expected scaling behavior $\Omega_{\chi} \sim m_{\chi}^{\gamma}$ with $\gamma=+2$. This behavior can be realized in a number of other ways as well.

How, then, can we "flip" this result and realize a scaling behavior in which $\Omega_{\chi} \sim m_{\chi}^{\gamma}$ with $\gamma<0$ ? Note that prior to utilizing the nonrelativistic approximation $E \approx m_{\chi}$ in the process of deriving Eq. (2.1), the powers of $m_{\chi}$ that appear in Eq. (2.1) had originally been powers of $E$. Thus, our interest is really in changing the powers of energy associated with the annihilation process. In particular, we are interested in finding a way to decrease the powers of energy in our expression for the abundance $\Omega_{\chi}$, or equivalently to increase the powers of energy in the cross-section $\langle\sigma v\rangle$.

Of course, one way of changing the powers of energy is already well known: if we imagine that the mediator $\phi$ has a nonzero mass $m_{\phi}$, then there is a natural process we may follow which amounts to replacing

$$
\frac{g_{\chi}^{2} g_{\psi}^{2}}{E^{2}} \longrightarrow G^{2} E^{2}
$$

where we have introduced the dimensionful effective coupling $G \equiv g_{\chi} g_{\psi} / m_{\phi}^{2}$. Indeed, this is nothing but the process of integrating out the mediator $\phi$-i.e., of taking $m_{\phi} \gg E \approx m_{\chi}$. This then leaves us with the effective annihilation process illustrated in Fig. 1(b). Note that the limit $m_{\phi} \gg m_{\chi}$ which underpins the integrating out of $\phi$ is opposite to the limit that yields the traditional result in 
Eq. (2.1). However, there is no conflict in doing this since we are no longer considering $m_{\phi}$ as parametrically tied to the weak scale.

As evident in Eq. (2.3), this process of integrating out $\phi$ results in a growing, unbounded cross-section whose unitarization was previously supplied through $\phi$. As a result, even for finite $m_{\phi}$, those dark-matter particles $\chi$ whose masses are significantly below $m_{\phi}$ experience an effective annihilation cross-section which grows rather than shrinks as a function of energy. For such dark-matter particles, standard thermal freeze-out then yields cosmological abundances which decrease, rather than increase, as a function of $m_{\chi}$.

To make these observations more explicit, let us imagine that we have an ensemble of dark-matter components $\chi_{i}$, $i=1, \ldots, N$, with masses $m_{i}$ satisfying $m_{i+1} \geq m_{i}$ for all $i \leq N-1$, whose annihilations are dominated by the process shown in Fig. 1(a) in which $\chi_{i}$ and its antiparticle $\bar{\chi}_{i}$ (which may or may not be identified with $\chi_{i}$ itself) annihilate into a pair of other, lighter particles $\psi$ and $\bar{\psi}$ through the exchange of a common mediator $\phi$. For concreteness we shall assume that $\chi_{i}$ and $\psi$ are Dirac fermions and that $\phi$ is a scalar. Here $\psi$ collectively denotes a SM state or a state in the dark sector which is lighter than the $\chi_{i}$; however, the identities of $\psi$ and $\bar{\psi}$ are not particularly important for determining the abundance spectrum, and we shall not specify their identities further. Likewise, we shall assume that the couplings between these fields take the simple forms $g_{\chi} \bar{\chi}_{i} \chi_{i} \phi$ and $g_{\psi} \phi \bar{\psi} \psi$ where $g_{\chi}$ and $g_{\psi}$ are arbitrary coupling constants. Note, in particular, that we are taking $g_{\chi}$ to be independent of $i$ and hence universal for the entire ensemble; other options will be briefly discussed in the Conclusions. Finally, we shall assume that $\phi$ and $\psi$ have arbitrary masses $m_{\phi}$ and $m_{\psi}$.

Given these assumptions, a straightforward calculation of the cross-section $\sigma_{i}$ for the process shown in Fig. 1(a) then yields the result

$$
\sigma_{i}=\frac{g_{\chi}^{2} g_{\psi}^{2}}{\pi(2 E)^{2}} \frac{\left(E^{2}-m_{i}^{2}\right)^{1 / 2}\left(E^{2}-m_{\psi}^{2}\right)^{3 / 2}}{\left(4 E^{2}-m_{\phi}^{2}\right)^{2}},
$$

where $E$ is the energy of each incoming $\chi$ particle in the center-of-mass frame. Because the dark matter is assumed nonrelativistic at the time of freeze-out, we may approximate $E^{2} \approx m_{i}^{2}\left(1+v^{2} / 4\right)$ where $v$ is the dark-matter relative velocity, whereupon we find that

$$
\sigma_{i} v=\frac{g_{\chi}^{2} g_{\psi}^{2}}{128 \pi m_{i}^{2}} v^{2} \frac{\left(1-m_{\psi}^{2} / m_{i}^{2}\right)^{3 / 2}}{\left(1-m_{\phi}^{2} / 4 m_{i}^{2}\right)^{2}} .
$$

Recognizing that $\left\langle v^{2}\right\rangle \sim T / m_{i}$ and that $T_{i} \approx m_{i} / 20$ up to logarithmic corrections, where $T_{i}$ is the freeze-out temperature of $\chi_{i}$, we then obtain the thermally averaged cross-section

$$
\left\langle\sigma_{i} v\right\rangle \sim \frac{g_{\chi}^{2} g_{\psi}^{2}}{m_{i}^{2}} \frac{\left(1-m_{\psi}^{2} / m_{i}^{2}\right)^{3 / 2}}{\left(1-m_{\phi}^{2} / 4 m_{i}^{2}\right)^{2}},
$$

where we are henceforth disregarding overall numerical factors.

For any such thermally averaged cross-section, the process of thermal freeze-out results in a present-day abundance given to leading order by [24-27]

$$
\Omega_{i} \approx \Omega_{\mathrm{CDM}}\left(\frac{\left\langle\sigma_{i} v\right\rangle}{1 \mathrm{pb}}\right)^{-1} e^{-\Gamma_{i} t_{\text {now }}}
$$

where $t_{\text {now }} \approx 13.8 \mathrm{Gyr}$ is the present age of the universe and where $\Gamma_{i}$ denotes the decay rate of the ensemble constituent $\chi_{i}$. Other than the $m_{i}$-dependence within $\sigma_{i}$ and $\Gamma_{i}$, this result is independent of $m_{i}$, up to logarithmic corrections. This expression for $\Omega_{i}$ is predicated on the assumption that $\chi_{i}$ is its own antiparticle; otherwise an additional overall factor of 2 would appear on the right side of this equation. For simplicity, we shall henceforth utilize the leading-order result in Eq. (2.7). We thus find that our resulting present-day cosmological abundance for this dark-matter component is given by

$$
\Omega_{i} \sim \frac{m_{i}^{2}}{g_{\chi}^{2} g_{\psi}^{2}} \frac{\left(1-m_{\phi}^{2} / 4 m_{i}^{2}\right)^{2}}{\left(1-m_{\psi}^{2} / m_{i}^{2}\right)^{3 / 2}} e^{-\Gamma_{i} t_{\text {now }}} .
$$

In the remainder of this paper, we shall focus on those ensemble constituents $\chi_{i}$ for which $\Gamma_{i} t_{\text {now }} \ll 1$-i.e., those components whose decays have a negligible effect on $\Omega_{i}$. We do this because these are precisely the ensemble components which survive today and whose abundances contribute to the present-day measurement of $\Omega_{\mathrm{CDM}}$. However, in this connection we remark that it is also possible to use the formalism we develop in this paper to study the abundances of those components whose lifetimes $\tau_{i} \equiv 1 / \Gamma_{i}$ are significantly shorter than $t_{\text {now }}$ and which have therefore already experienced significant decay prior to $t_{\text {now }}$. Indeed, for such components one natural approach would be to concentrate on the abundance $\Omega_{i}(t)$ of each component at $t=\tau_{i}$, as we expect such abundances to also obey the same types of inverse scaling relations that we expect for the abundances of those components surviving today [1]. However, comparing abundances at different times $\tau_{i}$ during cosmological history involves an additional complication. In particular, we must to take into account the differing abundance rescalings [1] that arise due to the differences between the decay time scales $\tau_{i}$ of the different ensemble constituents relative to $t_{\text {now }}$-time scales which may potentially even extend into different cosmological epochs.

Within our result in Eq. (2.8), it is natural to assume that $m_{i} \gg m_{\psi}$ for all $i$, as this is the condition that underpins the process $\bar{\chi}_{i} \chi_{i} \rightarrow \phi \rightarrow \bar{\psi} \psi$ which eventually induces the thermal freeze-out of $\chi_{i}$ and $\bar{\chi}_{i}$. We shall also assume that 
$\Gamma_{i} t_{\text {now }} \ll 1$, as described above. Under such circumstances, we then find that the dark-matter abundance scales with $m_{i}$ as

$$
\Omega_{i} \sim m_{i}^{2}\left(1-\frac{m_{\phi}^{2}}{4 m_{i}^{2}}\right)^{2} .
$$

In principle, there is no specified relationship between $m_{i}$ and $m_{\phi}$. However, we see from Eq. (2.9) that whether or not $\Omega_{i}$ increases or decreases with $m_{i}$ depends crucially on this relationship. In particular, we have the opposite limiting cases:

$$
\begin{array}{ll}
m_{i} \gg m_{\phi}: & \Omega_{i} \sim m_{i}^{2} \quad \Longrightarrow \gamma=+2, \\
m_{i} \ll m_{\phi}: & \Omega_{i} \sim m_{i}^{-2} \quad \Longrightarrow \gamma=-2 .
\end{array}
$$

Clearly the first case with an extremely light mediator $\phi$ yields the canonical scaling behavior that we already discussed in connection to the WIMP miracle. However, as promised, we see that the process of increasing the mediator mass $m_{\phi}$ and ultimately taking $m_{\phi} \gg m_{i}$ results in a flipping of the sign of the scaling exponent $\gamma$ from positive to negative values. Thus, in this regime, the cosmological abundances $\Omega_{i}$ resulting from thermal freeze-out decrease with increasing mass $m_{i}$-precisely as desired. Indeed, this result remains true for all ensemble constituents whose masses $m_{i}$ are significantly smaller than the intermediary mass $m_{\phi}$.

For dark-matter ensembles whose constituent masses are capped at some maximum value $m_{\max }$, taking $m_{\phi} \gg m_{\max }$ ensures that our desired scaling relationship holds across the entire dark-matter ensemble. Thus, in such cases, thermal freeze-out can indeed serve as a viable production mechanism within the DDM framework. However, in many theoretical constructions our resulting dark-matter ensemble contains an infinite number of constituents whose masses grow without bound. In such cases, our desired scaling behavior holds only across that (lighter) portion of the ensemble for which $m_{i} \ll m_{\phi}$. Indeed, as $m_{i}$ increases and becomes commensurate with $m_{\phi}$, other effects become relevant. For example, for constituents with $m_{i} \sim m_{\phi} / 2$, resonance effects become important. In this regime, provided that $\Gamma_{\phi}$ is not too small, we find that $\Omega_{i} \propto \Gamma_{\phi}^{2}$, where $\Gamma_{\phi}$ is the total width of the mediator. (For an extremely small mediator width, $\Omega_{i}$ is sensitive to the velocity distribution of $\chi_{i}$ and thus has a different parametric dependence [28,29]; moreover, energy-dependent corrections to $\Gamma_{\phi}$ can also have an effect on $\Omega_{i}$, as discussed in Ref. [30].) Finally, as $m_{i}$ increases even further, the corresponding abundances $\Omega_{i}$ ultimately begin to increase. Moreover, for $m_{i} \geq m_{\phi}$, annihilation to a pair of on-shell mediators becomes kinematically accessible. Since the corresponding cross-section does not have the same parametric dependence on $g_{\chi}, m_{i}$, and $m_{\phi}$ as in Eq. (2.6), our scaling relation for the abundances in Eq. (2.8) no longer holds in this regime.

Within most DDM models it is usually the lighter darkmatter constituents which play the most significant roles within the resulting dark-sector phenomenology. This is true for collider signatures $[3,4]$ as well as constraints coming from direct- and indirect-detection experiments [5-8]. Moreover, extremely heavy states within the ensemble may be expected to decay extremely rapidly in the early universe, potentially prior to the epochs during which such decays could run afoul of standard cosmological constraints and prior to the stage at which such states would experience thermal freeze-out. Thus, for most practical concerns, our main focus is usually on those lighter components of the ensemble which are most likely to survive to the present day and thus have the greatest phenomenological relevance. Fortunately, our mechanism for flipping the scaling of the abundance spectrum applies precisely for those dark-matter constituents. These issues will be discussed further in Sec. IV.

Thus, we conclude that thermal freeze-out can serve as a suitable abundance-production mechanism within the DDM framework. Indeed, within the annihilation channel we have considered here, we need only ensure that the mediator mass $m_{\phi}$ significantly exceeds $m_{i}$ over all relevant portions of the DDM ensemble. The mediator mass $m_{\phi}$ can then serve as a free parameter which may be adjusted so as to render $\Omega_{\text {tot }} \equiv \sum_{i} \Omega_{i}$ equal to $\Omega_{\mathrm{CDM}}$, as desired.

\section{GENERATING A SPECTRUM OF SCALING EXPONENTS: A MORE GENERAL STUDY}

Thus far, we have shown that we can flip the sign of the abundance scaling exponent $\gamma$ from +2 to -2 . This then produces a negative scaling exponent, consistent with our original goal. However, it is interesting for the purpose of considering many possible dark-sector phenomenologies and for general model-building purposes to explore the full range of values of $\gamma$ which may arise when our underlying annihilation process is varied. Moreover, even within the specific annihilation process we have considered, it is interesting to study more general cases beyond that in which $m_{\phi} / m_{i}$ is taken to infinity. Finally, as we shall see, there can also be final-state kinematic effects which we have thus far ignored but which might also potentially affect the values of the scaling exponent $\gamma$. We shall now undertake a general study of all of these possibilities.

Clearly, in order to obtain a variety of values of $\gamma$, one might consider a corresponding variety of dark-matter annihilation processes beyond that sketched in Fig. 1. Indeed, there is almost no limit to the complexity of annihilation processes which might be considered. However, it is also interesting to remain within the class of annihilation diagrams sharing the very natural topology of that in Fig. 1, but to consider alternative options for the spins of the internal and external particles as well as 
alternative Lorentz structures for the couplings between the dark and visible sectors. We shall follow the latter course in this paper.

Towards this end, let us reconsider the annihilation diagram in Fig. 1(a). We shall again consider a toy DDM model in which the annihilation rate for each ensemble constituent $\chi_{i}$ in the early universe is dominated by this $s$-channel process, and we shall again not make any assumptions concerning the specific identities of $\chi_{i}$ and $\psi$ except that $\psi$ is presumed lighter than $\chi_{i}$ for all $i$. We shall likewise not specify whether $\chi$ and $\psi$ are their own antiparticles. However, we shall now allow $\chi_{i}$ and $\psi$ to be either complex scalars or spin-1/2 fermions. Likewise, we shall allow $\phi$ to be either a spin-0 or spin-1 field. Furthermore, we shall allow the couplings between the mediator and the dark and visible sectors to have a variety of Lorentz structures: scalar (S), pseudoscalar $(\mathrm{P})$, vector $(\mathrm{V})$, or axial vector (A), constrained only as appropriate for the particle spins involved. In each case, we shall again assume that these couplings are the same for each ensemble constituent (and hence independent of the $i$ index), and in each case we shall again consider only the leading (renormalizable or super-renormalizable) operators.

The resulting possibilities are enumerated in Tables I and II. Note that the cases with spinless mediators coupled to spinless dark or visible matter give rise to superrenormalizable interactions; they thus depend on an arbitrary energy scale $\mu$. Moreover, unlike all other cases, those involving a spin-1 mediator and spinless dark or visible matter necessarily require derivative couplings. Finally, for logical consistency and completeness, we have included couplings involving the timelike components of vectorial interactions. However, these operators cannot couple to any external (initial or final) state regardless of the chargeconjugation, parity, or angular-momentum quantum numbers which that state might carry [31]. These couplings thus need not be considered further.

Also shown in Tables I and II are the values of certain corresponding indices $\left(\epsilon_{\chi}, \epsilon_{\psi}, r, s, t\right)$. The indices $\epsilon_{\chi}$ and $\epsilon_{\psi}$

TABLE I. Values of the indices $\epsilon_{\chi}$ and $r$ which correspond to different spins and coupling structures for the ensemble constituents $\chi_{i}$ and the mediator $\phi$.

\begin{tabular}{lcccc}
\hline \hline$\chi_{i}$ & $\phi$ & coupling & $\epsilon_{\chi}$ & $r$ \\
\hline spin-0 & spin-0 & S: $g_{\chi} \mu \chi^{*} \chi \phi$ & 1 & 0 \\
spin-1/2 & spin-0 & S: $g_{\chi} \bar{\chi} \chi \phi$ & 0 & 1 \\
spin-1/2 & spin-0 & P: $g_{\chi} \bar{\chi} \gamma_{5} \chi \phi$ & 0 & 0 \\
spin-0 & spin-1 (time) & V: $g_{\chi}\left(\chi^{*} \partial_{0} \chi\right) \phi^{0}$ & n/a & n/a \\
spin-0 & spin-1 (spatial) & V: $g_{\chi}\left(\chi^{*} \partial_{i} \chi\right) \phi^{i}$ & 0 & 1 \\
spin-1/2 & spin-1 (time) & V: $g_{\chi} \bar{\chi} \gamma_{0} \chi \phi^{0}$ & n/a & n/a \\
spin-1/2 & spin-1 (spatial) & V: $g_{\chi} \bar{\chi} \gamma_{i} \chi \phi^{i}$ & 0 & 0 \\
spin-1/2 & spin-1 (time) & A: $g_{\chi} \bar{\chi} \gamma_{0} \gamma_{5} \chi \phi^{0}$ & 0 & 0 \\
spin-1/2 & spin-1 (spatial) & A: $g_{\chi} \bar{\chi} \gamma_{i} \gamma_{5} \chi \phi^{i}$ & 0 & 1 \\
\hline \hline
\end{tabular}

TABLE II. Values of the indices $\epsilon_{\psi}, s$, and $t$ which correspond to different spins and coupling structures for the mediator $\phi$ and the particle species $\psi$ into which the ensemble constituents annihilate.

\begin{tabular}{lccccc}
\hline \hline$\phi$ & $\psi$ & coupling & $\epsilon_{\psi}$ & $s$ & $t$ \\
\hline spin-0 & spin-0 & $\mathrm{S}: g_{\psi} \mu \phi \psi^{*} \psi$ & 1 & 0 & 0 \\
spin-0 & spin-1/2 & $\mathrm{S}: g_{\psi} \phi \bar{\psi} \psi$ & 0 & 1 & 0 \\
spin-0 & spin-1/2 & $\mathrm{P}: g_{\psi} \phi \bar{\psi} \gamma_{5} \psi$ & 0 & 0 & 0 \\
spin-1 (time) & spin-0 & $\mathrm{V}: g_{\psi} \phi^{0}\left(\psi^{*} \partial_{0} \psi\right)$ & $\mathrm{n} / \mathrm{a}$ & $\mathrm{n} / \mathrm{a}$ & $\mathrm{n} / \mathrm{a}$ \\
spin-1 (spatial) & spin-0 & $\mathrm{V}: g_{\psi} \phi^{i}\left(\psi^{*} \partial_{i} \psi\right)$ & 0 & 1 & 0 \\
spin-1 (time) & spin-1/2 & $\mathrm{V}: g_{\psi} \phi^{0} \bar{\psi} \gamma_{0} \psi$ & $\mathrm{n} / \mathrm{a}$ & $\mathrm{n} / \mathrm{a}$ & $\mathrm{n} / \mathrm{a}$ \\
spin-1 (spatial) & spin-1/2 & $\mathrm{V}: g_{\psi} \phi^{i} \bar{\psi} \gamma_{i} \psi$ & 0 & 0 & 0 \\
spin-1 (time) & spin-1/2 & $\mathrm{A}: g_{\psi} \phi^{0} \bar{\psi} \gamma_{0} \gamma_{5} \psi$ & 0 & 0 & 1 \\
spin-1 (spatial) & spin-1/2 & $\mathrm{A}: g_{\psi} \phi^{i} \bar{\psi} \gamma_{i} \gamma_{5} \psi$ & 0 & 1 & 0 \\
\hline \hline
\end{tabular}

indicate the overall power of the energy scale $\mu$ that is needed in the corresponding coupling. (Equivalently, these indices are given by $4-d$ where $d$ is the mass dimension of the corresponding Lagrangian operator.) Likewise, we define $r=0$ if the mediator $\phi$ can couple to an initial state with total angular momentum $L=0$, and $r=1$ if the mediator can only couple to an initial state with $L=1$. Similarly, we define $s=0$ if $\phi$ can couple to a final state with $L=0$, and $s=1$ if $\phi$ can only couple to a final state with $L=1$. Finally, we define $t=1$ if the coupling between $\bar{\psi}, \psi$, and $\phi$ is chirality-suppressed, and $t=0$ otherwise. Note that if $\phi$ is a spin-1 particle with pseudovector couplings to both $\chi_{i}$ and $\psi$, contributions involving both the timelike and spacelike components of $\phi$ must be included. In cases in which $\psi$ is very light, the spacelike components yield the dominant contribution. By contrast, when $m_{\psi} \sim m_{i} / 2$, the timelike component dominates.

In order for the process $\bar{\chi}_{i} \chi_{i} \rightarrow \phi \rightarrow \bar{\psi} \psi$ to dominate the annihilation rate for each $\chi_{i}$, the contribution to that rate from coannihilation processes of the form $\bar{\chi}_{i} \chi_{j} \rightarrow \bar{\psi} \psi$ with $i \neq j$ must be suppressed. This occurs naturally, for example, in scenarios in which each of the $\chi_{i}$ is nontrivially charged under a different approximate symmetry. In addition, the collective contribution to the annihilation rate from intra-ensemble annihilation processes of the form $\bar{\chi}_{i} \chi_{i} \rightarrow \bar{\chi}_{j} \chi_{j}$, in which heavier ensemble constituents annihilate into lighter ones, must likewise be suppressed. This occurs naturally in scenarios in which $g_{\chi} \mu^{\epsilon_{\chi}} \ll g_{\psi} \mu^{\epsilon_{\psi}}$. However, as we shall see, a suppression of this sort arises in a variety of other contexts as well.

Given these assumptions concerning the nature of the dominant dark-matter annihilation processes, we can now calculate the corresponding cosmological abundances $\Omega_{i}$ that emerge after thermal freeze-out. For each spin/coupling combination in Tables I and II, the corresponding annihilation matrix elements $|\mathcal{M}|^{2}$, summed over final states and averaged over initial states, scale as 


$$
\begin{aligned}
|\mathcal{M}|^{2} \sim & g_{\chi}^{2} g_{\psi}^{2} v^{2 r}\left(\frac{\mu}{m_{i}}\right)^{2\left(\epsilon_{\chi}+\epsilon_{\psi}\right)} \\
& \times \frac{\left(1-m_{\psi}^{2} / m_{i}^{2}\right)^{s}}{\left(1-m_{\phi}^{2} / 4 m_{i}^{2}\right)^{2}}\left(\frac{m_{\psi}}{m_{i}}\right)^{2 t}
\end{aligned}
$$

where the corresponding values of $\epsilon_{\chi}, \epsilon_{\psi}, r, s$, and $t$ are listed in Tables I and II. The corresponding annihilation cross-sections then scale as

$$
\begin{aligned}
\sigma_{i} \sim & \frac{g_{\chi}^{2} g_{\psi}^{2}}{m_{i}^{2}} v^{2 r-1}\left(\frac{\mu}{m_{i}}\right)^{2\left(\epsilon_{\chi}+\epsilon_{\psi}\right)} \\
& \times \frac{\left(1-m_{\psi}^{2} / m_{i}^{2}\right)^{s+1 / 2}}{\left(1-m_{\phi}^{2} / 4 m_{i}^{2}\right)^{2}}\left(\frac{m_{\psi}}{m_{i}}\right)^{2 t} .
\end{aligned}
$$

Calculating $\left\langle\sigma_{i} v\right\rangle$ from these results is not difficult. As in Sec. II, we focus on the regime in which each $\chi_{i}$ freezes out at a temperature $T_{i}$ such that $x_{i} \equiv m_{i} / T_{i} \gg 3$. In this regime, the velocity distribution for each $\chi_{i}$ is already nonrelativistic, with speed $v \ll 1$, by the time freeze-out occurs. In this regime, the cross-section is reasonably well approximated by retaining the leading nonvanishing term in the series expansion

$$
\sigma_{i} v=a_{i}^{(0)}+a_{i}^{(1)} v^{2}+a_{i}^{(2)} v^{4}+\ldots
$$

in the quantity $v^{2}$. Recognizing that $\left\langle v^{2}\right\rangle \sim T / m_{i}$, we find that the corresponding thermal average at temperatures $T \sim T_{i}$ is given by [32]

$$
\left\langle\sigma_{i} v\right\rangle \approx a_{i}^{(0)}+\frac{3}{2} a_{i}^{(1)} x_{i}^{-1}+\frac{15}{8} a_{i}^{(2)} x_{i}^{-2}+\ldots
$$

Thus, in cases in which the annihilation is $s$-wave and the constant term $a_{i}^{(0)}$ dominates, the thermal average $\left\langle\sigma_{i} v\right\rangle$ scales with $m_{i}$ and $g_{i}$ across the ensemble in exactly the same way as $\sigma_{i} v$ itself. Moreover, since the ratio $x_{i}$ depends only logarithmically on $m_{i}$ and $g_{i}$ (due to the implicit dependence of $T_{i}$ on $m_{i}$ ), we find that even in cases in which $a_{i}^{(0)}=0$ and the annihilation is $p$-wave, $\left\langle\sigma_{i} v\right\rangle$ still scales with these parameters in approximately the same way as $\sigma_{i} v$, up to logarithmic corrections.

Given these results, we then find from Eq. (2.7) that our final abundances $\Omega_{i}$ scale across the ensemble as

$$
\Omega_{i} \sim \frac{m_{i}^{2}}{g_{\chi}^{2} g_{\psi}^{2}} m_{i}^{2\left(\epsilon_{\chi}+\epsilon_{\psi}+t\right)} \frac{\left(1-m_{\phi}^{2} / 4 m_{i}^{2}\right)^{2}}{\left(1-m_{\psi}^{2} / m_{i}^{2}\right)^{s+1 / 2}},
$$

where we continue to assume $\Gamma_{i} t_{\text {now }} \ll 1$ and thereby ignore the effects of particle decays, focusing instead on the original abundance produced by thermal freeze-out. In this expression, it is easy to understand the origins of each factor: (i) the leading factor of $m_{i}^{2}$ is nothing but the canonical contribution that already appeared in Eqs. (1.2) and (2.2);

(ii) the second factor $m_{i}^{2\left(\epsilon_{\chi}+\epsilon_{\psi}+t\right)}$ reflects the possibility of super-renormalizable couplings in Tables I and II, and also reflects the possibility of a chiralitysuppressed coupling between the mediator and the visible sector;

(iii) the third factor $\left(1-m_{\phi}^{2} / 4 m_{i}^{2}\right)^{2}$ reflects the contribution from the mediator; and

(iv) the final factor $\left(1-m_{\psi}^{2} / m_{i}^{2}\right)^{-s-1 / 2}$ reflects final-state kinematic effects.

If we consider only the first and third factors, we reproduce the result in Eq. (2.9). Indeed, we now see that it is legitimate to consider only the first and third factors in those cases for which $\epsilon_{\chi}=\epsilon_{\psi}=t=0$ (thereby eliminating the second factor) and for which $m_{i} \gg m_{\psi}$ for all $i$ (thereby eliminating the fourth factor). In such cases, we then reproduce our prior results in Eq. (2.10), with $\gamma$ flipping from +2 to -2 when the mediator is taken from extremely light to extremely heavy.

It is now apparent, however, that there are additional effects which can come into play. First, there is the contribution from the second factor. In general, the contribution from this factor increases the value of the scaling exponent by an amount

$$
\Delta \gamma \equiv 2\left(\epsilon_{\chi}+\epsilon_{\psi}+t\right) .
$$

It is immediately apparent from the various self-consistent coupling and mediator combinations in Tables I and II that the only allowed values for $\Delta \gamma$ are 0,2 , and 4 . We thus see that the possibility of super-renormalizable couplings and chirality-suppressed mediator/visible-sector couplings tends to drive $\gamma$ towards even more positive values. Indeed, for those combinations with $\Delta \gamma=4$, this effect completely cancels the effect from integrating out the mediator (i.e., the effect from taking $m_{\phi} \gg m_{i}$ for all $i$ ), restoring our positive traditional scaling exponent $\gamma=2$. However, the case with $\Delta \gamma=2$ leaves us with $\gamma=0$, producing cosmological abundances which are largely independent of the constituent masses $m_{i}$ to within the leading approximations we have been making. In such circumstances, it is then the subleading contributions to thermal freeze-out (coming perhaps from nondominant annihilation channels and subleading contributions to the thermal averaging process, etc.) which dictate the overall sign of the scaling exponent $\gamma$.

Finally, we consider the final-state kinematic effects coming from the fourth factor. When $m_{i} \gg m_{\psi}$ for all $i$, these effects are essentially independent of $m_{i}$ and thus do not alter the value of $\gamma$. Otherwise, when we merely have $m_{\min } \gtrsim m_{\psi}$ where $m_{\min }$ is the minimum of the $m_{i}$, this fourth factor enhances the cosmological abundances $\Omega_{i}$ but does so increasingly weakly as a function of the constituent mass $m_{i}$. In other words, this factor is greater than 1 but 
decreases as a function of $m_{i}$. This then tends to provide a negative (although $m_{i}$-dependent) contribution to $\gamma$ which can again pull the overall value of the scaling exponent $\gamma$ towards negative values.

We thus see that the question of whether the resulting values of $\gamma$ are positive or negative depends on the balancing between a number of factors governing the annihilation process. The canonical factor gives a contribution $\gamma=2$, and the coupling and chirality factors make a potential additional positive contribution given in Eq. (3.6). By contrast, integrating out the mediator $\phi$ tends to decrease the value of $\gamma$ by four units, as we have seen in Sec. II, and this is then further decreased by the final-state kinematic effects.

Thus far, we have treated $\gamma$ as if this scaling exponent were constant across the entire ensemble. In other words, we have implicitly assumed that $\Omega_{i}$ exhibits a pure powerlaw dependence on $m_{i}$. This is certainly true for the contribution from the canonical first factor in Eq. (3.5), and true even for the extra contribution in Eq. (3.6) coming from the second factor. It is also true for the third factor as long as we consider the mediator $\phi$ to be either extremely light $\left(m_{\phi} \ll m_{i}\right.$ for all $\left.i\right)$ or extremely heavy $\left(m_{\phi} \gg m_{i}\right.$ for all $i$ ), and it is trivially true even for the fourth factor as long as we consider our final-state particles to also be extremely light, with $m_{\psi} \ll m_{i}$ for all $i$. However, the scaling-exponent contributions from the third and fourth factors in Eq. (3.5) are generally $m_{i}$-dependent, which means that our total scaling exponent $\gamma$ will also be $m_{i}$-dependent. Indeed, recognizing this fact is critical if we wish to extend our analysis beyond the limiting approximations outlined above.

Fortunately, it is not difficult to obtain the corresponding results for these mass-dependent scaling exponents $\gamma(m)$. In general, we have seen from Eq. (3.5) that $\Omega(m)$ can be viewed as a continuous function which varies with the mass scale $m$ within the allowed range $m_{\psi}<m<m_{\phi} / 2$ according to

$$
\Omega(m) \sim \frac{m^{2}}{g_{\chi}^{2} g_{\psi}^{2}} m^{2\left(\epsilon_{\chi}+\epsilon_{\psi}+t\right)} \frac{\left(1-m_{\phi}^{2} / 4 m^{2}\right)^{2}}{\left(1-m_{\psi}^{2} / m^{2}\right)^{s+1 / 2}} .
$$

Given this, we can define our effective scaling exponent at any value of $m$ via the relation $\Omega(m) \sim m^{\gamma}$, or equivalently

$$
\gamma(m) \equiv \frac{d \ln \Omega(m)}{d \ln m}=\frac{m}{\Omega(m)} \frac{d \Omega(m)}{d m} .
$$

For the abundance in Eq. (3.7), we then find

$$
\gamma(m)=2+\Delta \gamma+\frac{1}{m^{2} / m_{\phi}^{2}-1 / 4}+\frac{2 s+1}{1-m^{2} / m_{\psi}^{2}},
$$

where $\Delta \gamma$ is given in Eq. (3.6). Indeed, the separate terms in Eq. (3.9) are the contributions from the corresponding factors in Eq. (3.7).
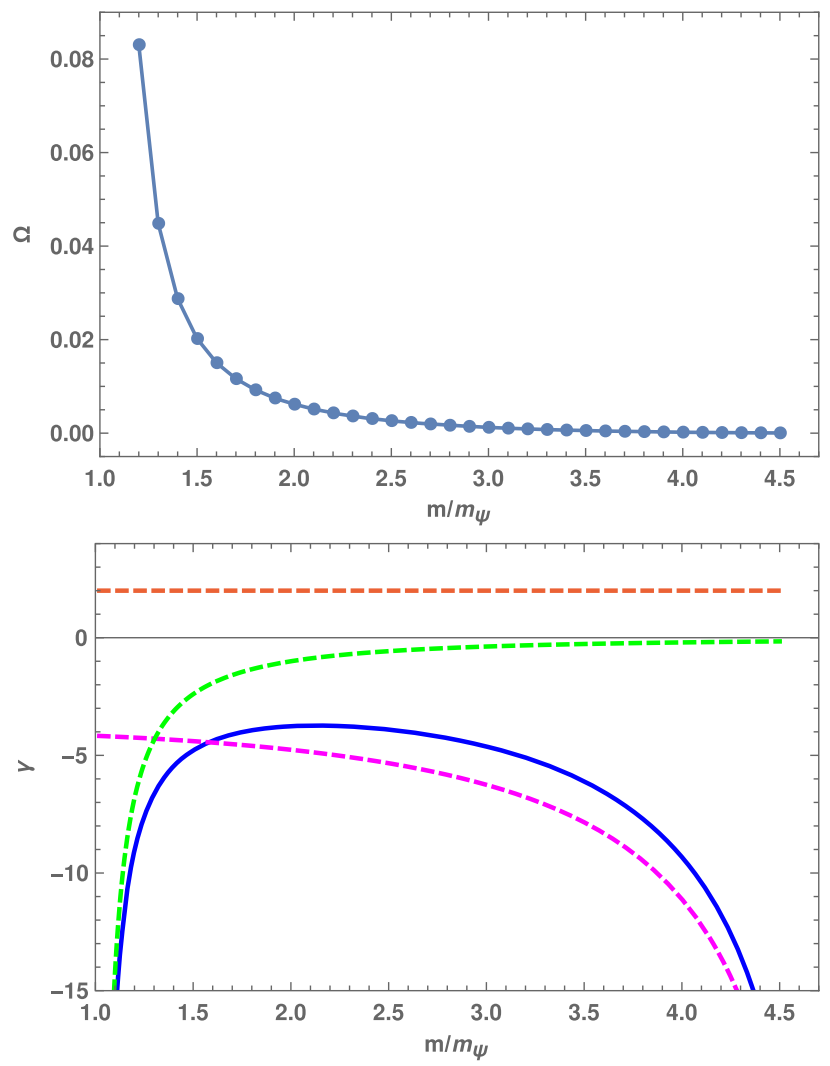

FIG. 2. Abundances $\Omega_{i}$ (upper panel) and contributions to the corresponding scaling exponent $\gamma$ (lower panel) for $\epsilon_{\chi}=\epsilon_{\psi}=$ $r=t=0$ and $s=1$. We have taken $m_{\phi}=10 m_{\psi}$ and assumed a mass spectrum $m_{i} / m_{\psi}=\{1.2,1.3,1.4, \ldots, 4.5\}$. The corresponding values of $\Omega_{i}$ have been normalized so that $\sum_{i} \Omega_{i}=$ $\Omega_{\mathrm{CDM}} \approx 0.26$. We see from the upper panel that $\Omega_{i}$ indeed falls as a function of $m_{i}$, as desired, even though these abundances arise from thermal freeze-out. The solid blue curve in the lower panel indicates the corresponding scaling exponent $\gamma(m)$, which is negative throughout the appropriate dark-matter mass range. This curve receives additive contributions from the canonical contribution $\gamma=+2$ (red dashed line), the effects of the heavy mediator $\phi$ (magenta dashed curve), and the effects of final-state kinematics (green dashed curve). Given that the canonical contribution alone leads to $\gamma=+2$, we see that the contributions from the latter two effects combine to pull this result into the $\gamma<0$ range and also to introduce a nontrivial mass-dependence for $\gamma(m)$.

In Fig. 2 we have plotted the results for $\Omega(m)$ and $\gamma(m)$ as functions of $m$ over the mass range $m_{\psi}<m<m_{\phi} / 2$. For these plots we have taken $m_{\phi}=10 m_{\psi}$. Within this mass range, we have also chosen a discrete mass spectrum $m_{i} / m_{\psi}=\{1.2,1.3,1.4, \ldots, 4.5\}$, and we have normalized the corresponding abundances $\Omega_{i}$ [and thus the overall abundance curve $\Omega(m)$ on which these abundances lie] so that $\Omega_{\mathrm{tot}} \equiv \sum_{i} \Omega_{i}=\Omega_{\mathrm{CDM}} \approx 0.26$. We have also taken our underlying annihilation process to have $\epsilon_{\chi}=\epsilon_{\psi}=r=$ $t=0$ and $s=1$. Note that our choice of a particular discrete constituent mass spectrum $\left\{m_{i}\right\}$ populating the 
allowed mass range $m_{\psi}<m<m_{\phi} / 2$ allows us to normalize our total cosmological abundance curve $\Omega(m)$ and thereby determine a particular partitioning of $\Omega_{\mathrm{CDM}}$ across the different contributions $\Omega_{i}$. Our choice of the discrete mass spectrum $\left\{m_{i}\right\}$ along these curves otherwise plays no essential role in fixing the behavior of $\Omega(m)$ as a function of $m$.

We see from the upper panel of Fig. 2 that the ensemble abundances $\Omega_{i}$ fall as a function of the constituent masses $m_{i}$, as desired, even though these abundances arise from thermal freeze-out. This then verifies explicitly that thermal freeze-out can yield cosmological abundances which decrease, rather than increase, as a function of the mass of the individual dark-matter constituents. Indeed, this behavior is smooth and resembles the behavior that has been observed for other nonthermal abundance-production mechanisms.

The lower panel of Fig. 2 illustrates the corresponding behavior of the scaling exponent function $\gamma(m)$ in Eq. (3.9). The solid blue curve in the lower panel indicates the total scaling exponent $\gamma(m)$, which is negative throughout the appropriate dark-matter mass range. Indeed, this curve receives additive contributions from the canonical result $\gamma=+2$ (red dashed line), the effects of the heavy mediator $\phi$ (magenta dashed curve), and the effects of final-state kinematics (green dashed curve). These are respectively the first, third, and fourth terms in Eq. (3.9). As already noted, we see that the contributions from the latter two effects combine to overwhelm the canonical contribution $\gamma=+2$ and pull the resulting scaling exponent into the $\gamma<0$ range. They also introduce a nontrivial mass-dependence for $\gamma$.

In the limit in which $m_{\phi} \rightarrow \infty$ (so that the mediator is fully integrated out of the theory), the magenta curve starts at $\gamma=-4$ and remains essentially flat. Likewise, within mass regions for which $m \gg m_{\psi}$, the final-state kinematic effects disappear and the green curve also becomes essentially flat at $\gamma=0$. Thus, in these limits, we see that our canonical contribution $\gamma=+2$ is uniformly pulled down by the mediator effects to $\gamma=-2$, as discussed in Sec. II. However, we now see that for masses $m$ which are not that far below $m_{\phi} / 2$ or not that far above $m_{\psi}$ (i.e., for masses at the lighter and heavier ends of the allowed mass range), the net effects of the mediator and the final-state kinematics are to bend the $\gamma(m)$ blue curve further below $\gamma=-2$. This enhances the rates at which the corresponding abundances fall as functions of the mass. Indeed, with both effects together, we find that the maximum value of $\gamma$ plotted in the lower panel of Fig. 2 is approximately -4 rather than the value $\gamma=-2$ that would have existed without these effects. Thus even the behavior of the central portion of the darkmatter ensemble is altered by these effects.

It is important to note that while the specific choice of the discrete constituent mass spectrum $\left\{m_{i}\right\}$ within our overall allowed mass range has no effect on the behavior of the scaling exponents $\gamma(m)$, this choice can nevertheless

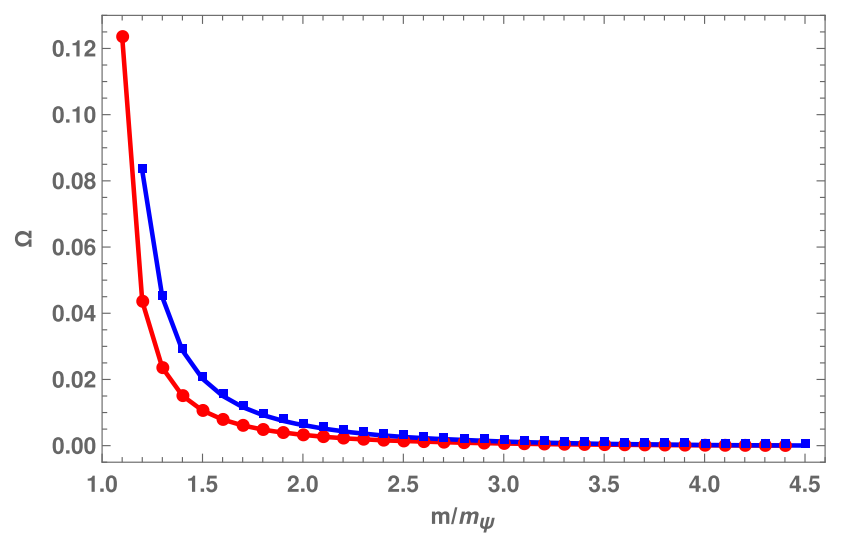

FIG. 3. Abundances $\Omega_{i}$ with $s=1, \epsilon_{\chi}=\epsilon_{\psi}=r=t=0$, and $m_{\phi} / m_{\psi}=10$. The blue curve is the same as in the top panel of Fig. 2, corresponding to the discrete constituent mass spectrum $m_{i} / m_{\psi}=\{1.2,1.3,1.4, \ldots, 4.5\}$, while the red curve corresponds to the same mass spectrum shifted downward by $\Delta m_{i} / m_{\psi}=$ -0.1 . In each case the corresponding values of $\Omega_{i}$ have been normalized so that $\sum_{i} \Omega_{i}=\Omega_{\mathrm{CDM}} \approx 0.26$. As a result of the falling behavior of $\Omega_{i}$ as a function of mass $m_{i}$, we see that the downward shift of our spectrum induces a renormalization of all of the states and alters the magnitudes of the relative contributions to $\Omega_{\mathrm{CDM}}$, shifting the abundance distribution of the resulting DDM ensemble from one with $\eta \approx 0.65$ (blue) to one with $\eta \approx 0.52$ (red).

significantly affect the relative partitioning of the total darkmatter abundance $\Omega_{\mathrm{CDM}}$ across the ensemble. For example, in Fig. 3 we have plotted the abundances for two different mass spectra: that already plotted in the top panel of Fig. 2 (blue), and the spectrum that results by shifting the mass of each component downward by $\Delta m_{i} / m_{\psi}=-0.1$ (red). In each case, we have normalized the corresponding abundances $\Omega_{i}$ [and thus the corresponding general $\Omega(m)$ curve] so as to hold the total abundance $\Omega_{\mathrm{tot}} \equiv \sum_{i} \Omega_{i}=\Omega_{\mathrm{CDM}} \approx$ 0.26 fixed. Two observations are immediately apparent. First, the blue curve is the same as the red curve except for an overall multiplicative factor (which in this case is approximately 1.905). Second, however, the magnitudes of the relative constituent contributions $\Omega_{i}$ to the total abundance $\Omega_{\text {tot }}$ are nontrivially altered due to the change in discrete masses. One useful way to characterize the abundance distribution of a given DDM ensemble is through the so-called "tower fraction" $\eta$, defined as $[1,2]$

$$
\eta \equiv 1-\frac{\Omega_{\max }}{\sum_{i} \Omega_{i}} \quad \text { where } \Omega_{\max } \equiv \max _{i} \Omega_{i}
$$

Note that $0 \leq \eta<1$. In general, the value of $\eta$ indicates how much of the total abundance of the ensemble is carried by those states which are not the dominant one. Thus smaller values of $\eta$ correspond to the more traditional ensembles in which only one or a few components carry the bulk of $\Omega_{\mathrm{CDM}}$, while larger values of $\eta$ correspond to more 
DDM-like ensembles in which the abundance is more generally distributed across the ensemble. We see from the abundances plotted in Fig. 3 that the blue curve corresponds to approximately $\eta \approx 0.65$, while the red curve corresponds to approximately $\eta \approx 0.52$. Thus, for abundances $\Omega_{i}$ which fall as a function of mass, shifting the spectrum of discrete constituent masses towards higher masses tends to increase the value of $\eta$ and thereby enhance the DDM-like nature of the corresponding ensemble.

Thus far we have only considered the case with $\epsilon_{\chi}=$ $\epsilon_{\psi}=t=0$. Thus $\Delta \gamma=0$, and the second factor in Eq. (3.9) has no effect. Likewise, we have only considered the case with $m_{\phi} / m_{\psi}=10$, which is a relatively small mass hierarchy. In principle-and for phenomenological purposes-we are interested in much larger values of this ratio. We therefore turn to examine how the basic picture outlined above changes for $\Delta \gamma>0$ and for larger values of $m_{\phi} / m_{\psi}$.

It is readily apparent that choosing spin and coupling structures in Tables I and II with nonzero values of $\left\{\epsilon_{\chi}, \epsilon_{\psi}, t\right\}$ only serves to shift the $\gamma$-curves in Fig. 2 uniformly upwards by an amount $\Delta \gamma$. For $\Delta \gamma=2$, it is clear that the blue curve remains completely within the $\gamma<0$ range. Thus, for $\Delta \gamma=2$ and for the value of $m_{\phi} / m_{\psi}=10$ chosen for the plots in Fig. 2, the abundances $\Omega_{i}$ continue to scale inversely with the dark-matter masses $m_{i}$ throughout the ensemble. However, when shifted by $\Delta \gamma=+4$-as occurs when the couplings of the mediator to the dark and visible sectors are both superrenormalizable, with $\epsilon_{\chi}=\epsilon_{\psi}=1$ - the blue curve within the lower panel of Fig. 2 actually exceeds zero within the approximate region $1.3 \lesssim m / m_{\psi} \lesssim 2.7$. This means that the corresponding cosmological abundances $\Omega_{i}$ fall as a function of $m_{i}$ for $m_{i} / m_{\psi} \lesssim 1.3$ and then rise for $1.3 \lesssim m_{i} / m_{\psi} \lesssim 2.7$ before ultimately falling again for $m_{i} / m_{\psi} \gtrsim 2.7$.

The behavior of the cosmological abundance $\Omega(m)$ as a function of $m$ is shown in Fig. 4 for $\Delta \gamma=0,2$, and 4. The upper panel shows the full range of cosmological abundances realized in these scenarios, while the lower panel shows the lower region of the upper panel in more detail. For these plots, we have taken $m_{\phi} / m_{\psi}=10$ and $s=0$. We have also assumed the discrete mass spectrum $m_{i} / m_{\psi}=$ $1.1,1.2, \ldots, 4.5$ and plotted the corresponding values of $\Omega_{i}$. In each case, an overall normalization has been chosen so that $\Omega_{\text {tot }}=\Omega_{\mathrm{CDM}} \approx 0.26$. The blue curve in the upper panel (representing the $\Delta \gamma=0$ case) can be compared with the red curve in Fig. 3 in order to discern the effects of taking $s=0$ rather than $s=1$. However, as expected, we now see that increasing $\Delta \gamma$ has the net effect of decreasing the rate at which the corresponding abundances $\Omega_{i}$ fall as functions of $m$. Indeed, increasing $\Delta \gamma$ all the way to 4 even manages to induce a localized mass region in which the abundances $\Omega_{i}$ actually increase as a function of $m$. Thus, for $\Delta \gamma=4$, we see that the effects from both a heavy mediator and finalstate kinematics have conspired to produce not only a
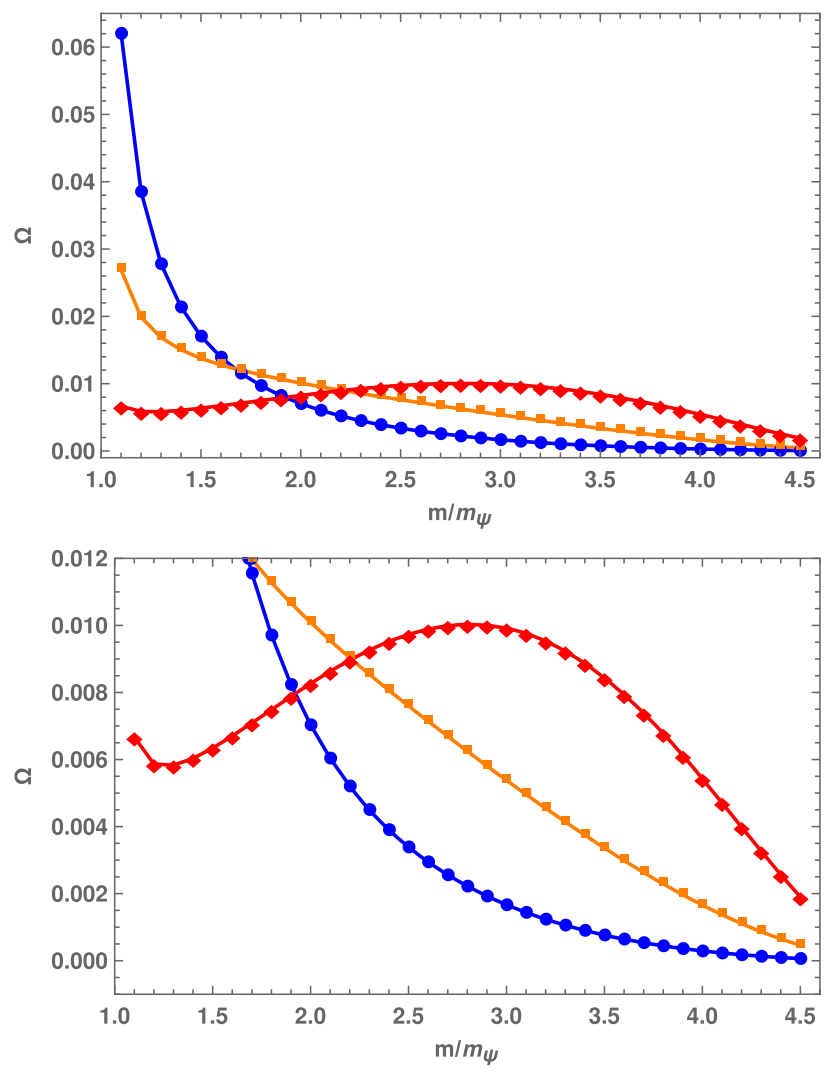

FIG. 4. Upper panel: same as the upper panel of Fig. 2 except with $s=0$, plotted for different values of $\Delta \gamma$. The cases with $\Delta \gamma=0,2,4$ correspond to the blue, orange, and red curves, respectively. As in the upper panel of Fig. 2, each curve is individually normalized so that the total abundance $\sum_{i} \Omega_{i}$ is fixed at $\Omega_{\mathrm{CDM}} \approx 0.26$. Lower panel: a zoom-in of the small-abundance portion of the upper panel. We see that the abundance curves are monotonically decreasing as a function of $m$ for $\Delta \gamma=0$ and $\Delta \gamma=2$, while for $\Delta \gamma=4$ there is a region $1.3 \lesssim m / m_{\psi} \lesssim 2.7$ over which the abundances increase as a function of $m$ before decreasing again.

nonmonotonic $\Omega(m)$ function but, with it, also a local minimum for $\Omega(m)$, as shown in Fig. 4. This could thereby give rise to a potentially interesting new phenomenology. Indeed, in such cases we see that thermal freeze-out has effectively selected a particular mass scale for special treatment, endowing the corresponding member of the dark-matter ensemble with a small, extra bit of cosmological invisibility as compared with its immediate lighter and heavier neighbors.

Finally, we consider the behavior that emerges for larger hierarchies $m_{\phi} / m_{\psi}$. It is important to stress that this is not the same as integrating out the mediator $\phi$, since we are still considering all possible values of $m / m_{\phi}<1 / 2$ without requiring $m \ll m_{\phi}$. In order to make meaningful comparisons with different rescaled values of $m_{\phi} / m_{\psi}$, we simultaneously rescale the mass differences across our assumed discrete mass spectrum. In other words, the discrete mass 

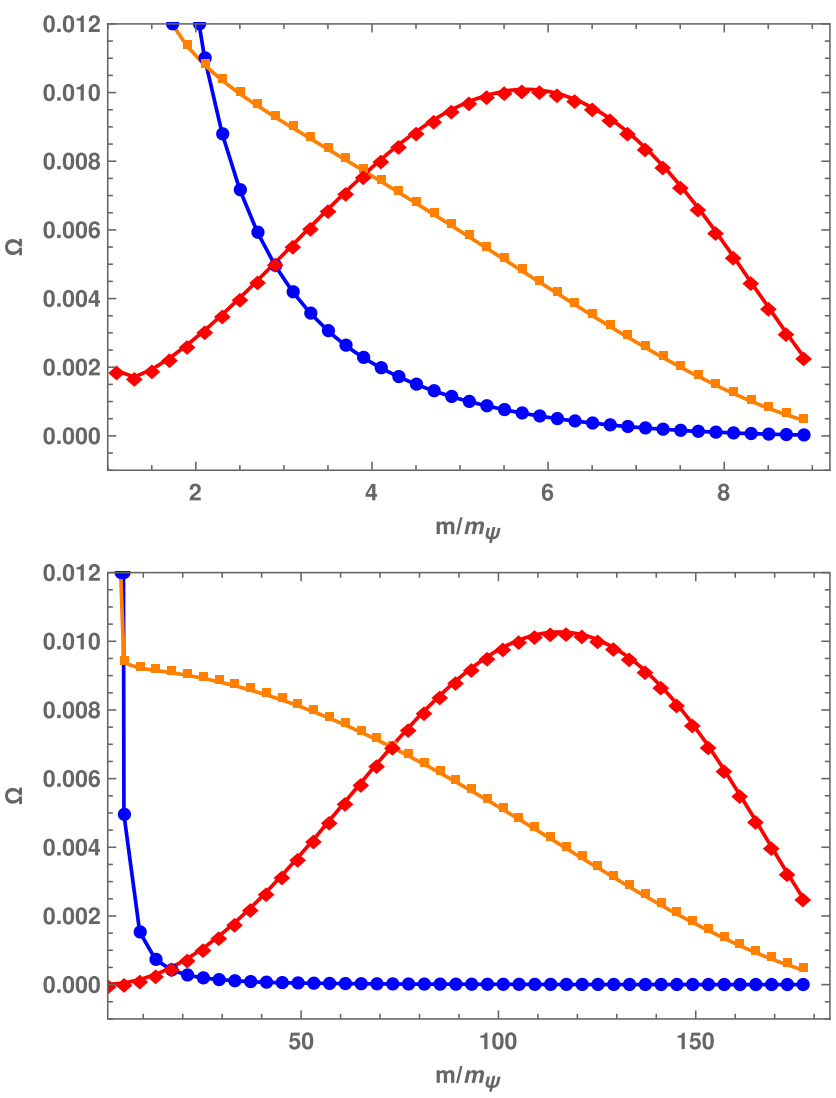

FIG. 5. Same as the lower panel of Fig. 4, except with $m_{\phi} / m_{\psi}$ now increased to $m_{\phi} / m_{\psi}=20$ (upper panel) and $m_{\phi} / m_{\psi}=400$ (lower panel), with our discrete constituent mass spectrum rescaled accordingly. As $m_{\phi} / m_{\psi}$ increases, the $\Delta \gamma=4$ curves (red) exhibit increasingly pronounced local minima and maxima while the $\Delta \gamma=0,2$ curves (blue and orange) remain monotonically decreasing. The behavior of the curves shown in the lower panel essentially illustrates the behavior that emerges in the asymptotic $m_{\phi} / m_{\psi} \rightarrow \infty$ limit. Thus we see that for all values of $\Delta \gamma$ and $m_{\phi} / m_{\psi}$ there exist relatively large (and occasionally unconstrained) regions of $m$ over which the corresponding cosmological abundances $\Omega$ fall as a function of $m$.

spectrum $\left(m_{k}-m_{0}\right) / m_{\psi}=0.1 k$ with $m_{0} \equiv 1.1 m_{\psi}$ that we previously took for $m_{\phi} / m_{\psi}=10$ will now be taken as $\left(m_{k}-m_{0}\right) / m_{\psi}=0.01\left(m_{\phi} / m_{\psi}\right) k$ for any value of $m_{\phi} / m_{\psi}$. This reduces to the original mass spectrum for $m_{\phi} / m_{\psi}=10$ but otherwise scales so as to similarly fill the allowed range $m_{\psi} \leq m \leq m_{\phi} / 2$ while keeping the lightest component anchored at $m_{0}=1.1 m_{\psi}$.

The resulting cosmological abundances are shown in Fig. 5 for $m_{\phi} / m_{\psi}=20$ (upper panel) and $m_{\phi} / m_{\psi}=400$ (lower panel). As in Fig. 4, we have once again taken $s=0$. In general, for $\Delta \gamma=4$, we see that increasing the value of $m_{\phi} / m_{\psi}$ tends to enhance the nonmonotonicity of the abundance $\Omega(m)$ as a function of $m$ that we have already observed in Fig. 4. By contrast, the cases with $\Delta \gamma \leq 2$ remain completely monotonic.
This behavior survives even as $m_{\phi} / m_{\psi} \rightarrow \infty$. Indeed, for $m_{\phi} / m_{\psi} \rightarrow \infty$, we find that the cosmological abundances monotonically decrease as a function of $m$ for $\Delta \gamma=0,2$. By contrast, for any $\Delta \gamma>2$, we find that these abundances $\Omega(m)$ monotonically decrease as a function of $m$ within only two disconnected regions: a very small region at low masses near $m_{\psi}$ given by

$$
\frac{m}{m_{\psi}}<\sqrt{1+\frac{2 s+1}{\Delta \gamma-2}},
$$

and a significantly larger region at higher masses given by

$$
\frac{m}{m_{\phi}}>\sqrt{\frac{1}{4}-\frac{1}{2+\Delta \gamma}} .
$$

For other ranges of $m$, the corresponding abundances decrease as a function of $m$.

This nonmonotonic behavior gives great flexibility to the DDM model-builder: one need only select a model with ensemble constituent masses $m_{i}$ at appropriate locations along these curves in order to endow these constituents with cosmological abundances which either rise or fall with mass, at will. For example, if one wishes to have ensemble constituents whose cosmological abundances all fall monotonically with mass, one need only choose these constituents to have masses $m_{i}$ within the ranges specified above (or choose $\Delta \gamma \leq 2$, for which the corresponding ranges are unrestrained). Thus even the cases with $\Delta \gamma>2$ are capable of yielding purely monotonically falling abundances $\Omega_{i}$, as typically desired for DDM. However, the presence of nonmonotonicities in these cases also allows for other possibilities. For example, through appropriate choices of constituent masses $m_{i}$, one can imagine situations in which thermal freeze-out yields growing abundances for one darkmatter species (or one portion of a DDM ensemble) and yet decreasing abundances for another. Indeed, by adjusting the values of the constituent masses $m_{i}$, one can even dial the relevant value of the scaling exponent $\gamma$ in a continuous way. These observations thus significantly enrich the phenomenological possibilities for DDM model-building.

Thus far, we have considered the cases in which our final-state particles $\psi$ are spin- 0 or spin- $1 / 2$. However, it is also useful to consider the case in which $\psi$ is a spin-1 particle, henceforth to be denoted $A^{\mu}$. For concreteness, let us imagine that $A^{\mu}$ is the gauge boson of a (potentially broken) gauge symmetry, either Abelian or non-Abelian, and let us endow $A^{\mu}$ with an arbitrary mass $m_{\psi}=m_{A}$. For concreteness, let us further take $\chi$ to be spin-1/2, our mediator $\phi$ to be spin- 0 , and our $\phi A_{\mu} A^{\mu}$ coupling to be of the form that emerges from the gauge-invariant operator

$$
\frac{c_{A}}{\Lambda} \phi F_{\mu \nu}^{a} F^{\mu \nu a}
$$


Note that we adopt this gauge-invariant coupling structure in order to accommodate the case with $m_{A}=0$, for which our gauge symmetry is necessarily unbroken. Calculations similar to those above then lead to a cosmological abundance $\Omega(m)$ whose scaling behavior takes the form given in Eq. (3.7) with $s=t=0$, with $g_{\psi} \rightarrow c_{A}$ and $\epsilon_{\psi} \rightarrow \epsilon_{A}=-1$, and with the final-state kinematic factor $\left(1-m_{\psi}^{2} / m^{2}\right)^{-1 / 2}$ now multiplied by an additional kinematic factor $f(x) \equiv$ $\left(1-x^{2}+3 x^{4} / 8\right)^{-1}$ where $x \equiv m_{A} / m$. For $x \ll 1$, we can approximate $f(x) \approx\left(1-x^{2}\right)^{-1}$, which is tantamount to our traditional form in Eq. (3.7) with $s=1$ rather than $s=0$. But regardless of the value of $x$, we see that the primary effect of taking our final-state particles to be spin-1 with the coupling indicated in Eq. (3.13) is that $\epsilon_{\psi}$ (now denoted $\epsilon_{A}$ ) is negative. This is a direct consequence of the fact that the leading-order gauge-invariant coupling between a spin-0 mediator and two vector fields, as in Eq. (3.13), is nonrenormalizable. This then has the net effect of allowing situations with $\Delta \gamma=-2$, which only further strengthens the desired inverse scaling between the abundances and masses and which produces values for $\gamma$ which are even more negative than those which emerge for any other cases considered thus far.

If we restrict our attention to cases in which the vectors $A^{\mu}$ are necessarily massive, then the gauge symmetry is necessarily broken and a fully gauge-invariant coupling such as that in Eq. (3.13) is not required. In such cases, we may instead consider a direct super-renormalizable coupling of the form

$$
c_{A} \mu \phi A_{\mu}^{a} A^{\mu a},
$$

which is reminiscent of the couplings for massive vector mediators in Tables I and II. We then find that the corresponding cosmological abundance $\Omega(m)$ varies with $m$ exactly as it does for the $\phi F F$ coupling discussed above, except with $f(x)$ now given by $f(x) \equiv\left(1-x^{2}+3 x^{4} / 4\right)^{-1}$. Thus, for all intents and purposes, the change of coupling structure from that in Eq. (3.13) to that in Eq. (3.14) has very little effect on the resulting scaling of $\Omega$ with $m$. At first glance, it may seem surprising that we continue to have $\epsilon_{A}=$ -1 when we are now dealing with the super-renormalizable operator in Eq. (3.14). However, we can always algebraically recast our result into a form with $\epsilon_{A}=+1$ by replacing $f(x)$ with $g(x) \equiv\left(3 x^{4} / 4\right) f(x)=\left(1-4 x^{-2} / 3+4 x^{-4} / 3\right)^{-1}$. Of course, this algebraic manipulation does not change the underlying scaling behavior, which continues to be the same as that for the $\phi F F$ coupling. We see, then, that the abundance function $\Omega(m)$ resulting from the coupling in Eq. (3.14) has an almost identical scaling behavior as that resulting from the coupling in Eq. (3.13). Indeed, in both cases the resulting scaling exponents $\gamma$ are more negative than for any other cases we have considered.

\section{BALANCING LIFETIMES AGAINST ABUNDANCES: GENERAL CONSTRAINTS FOR DDM VIABILITY}

In Secs. II and III, we have discussed the means by which we can achieve an ensemble of states for which the cosmological abundances produced through thermal freeze-out scale inversely with mass. As discussed in the Introduction, and as we shall further discuss below, this scaling behavior is a primary ingredient leading to a viable DDM ensemble.

However, this alone is not sufficient. The DDM framework also requires certain scaling behaviors for the decay widths $\Gamma_{i}$ of our ensemble constituents, where we assume that the dominant decay mode of each DDM ensemble constituent is directly into SM states. Likewise, the DDM framework also requires certain scaling relations for the mass distribution of states across the ensemble, or equivalently for the corresponding ensemble density of states. Indeed, what ultimately matters for the phenomenological viability of a DDM ensemble is how these different scaling behaviors balance against each other [1]. In this section we shall briefly review the scaling relations for the decay widths and densities of states. We shall also outline some of the general constraints that they must satisfy, and what our results for the scaling behaviors of the cosmological abundances imply about these other scaling relations.

In general, a given ensemble of dark-matter states will typically have SM decay widths $\Gamma_{i}$ exhibiting simple scaling behaviors as functions of the constituent masses $m_{i}$. For example, let us consider what is perhaps the simplest decay pattern in which each ensemble constituent $\chi_{i}$ decays directly into two final-state particles $f$ and $\bar{f}$ whose masses are well below those of the constituents: $m_{f} \ll m_{i}$ for all $i$. We also assume that this decay occurs through a dimension- $d$ contact operator which therefore takes the form $\mathcal{O}_{i} \sim c \chi_{i} \bar{f} f / \Lambda^{d-4}$ where $\Lambda$ is an appropriate mass scale. Since the matrix element $\mathcal{M}$ for a $1 \rightarrow 2$ decay of this form must have mass dimension +1 , we then find through elementary dimensional analysis that the matrix element must scale with the constituent mass as $\mathcal{M} \sim m_{i}^{d-3}$. Since the decay width under such circumstances generally scales as $\Gamma_{i} \sim|\mathcal{M}|^{2} / m_{i}$, we then find that

$$
\Gamma_{i} \sim m_{i}^{2 d-7} .
$$

Thus the decay widths scale as a positive power of the mass for $d \geq 4$, but with a negative power for $d \leq 3$. In general, we can write our decay-width scaling relation in the form

$$
\Gamma_{i} \sim m_{i}^{y}
$$

where $y$ is an appropriate scaling exponent. Of course, for decays of the simple form described above, we have $y=2 d-7$. 
It is also natural to consider ensembles of states in which the distribution of constituent masses $m_{i}$, and thus the corresponding effective densities of states $n_{m}$ per unit mass, also obey power-law scaling relations. For example, in many concrete realizations of DDM ensembles one finds that the constituent masses are distributed as

$$
m_{k} \sim k^{\delta}
$$

where $\delta>0$ is another scaling exponent. Special benchmark cases include $\delta=1$ (corresponding to the KK spectrum $m_{k} \sim k / R$ resulting from compactification on a circle or orbifold of radius $R[1,2,11])$ as well as $\delta=1 / 2$ (corresponding to the spectrum $\alpha^{\prime} m_{k}^{2} \sim k$ of string excitations [12,14], where $\alpha^{\prime}$ is the Regge slope, i.e., the inverse of the squared string scale, and where $k$ is the string excitation number). A general scaling relation of the form in Eq. (4.3) for the ensemble mass spectrum then implies a corresponding density of states $n_{m}$ per unit mass which scales with mass as

$$
n_{m} \sim m^{1 / \delta-1}
$$

We thus have three independent scaling relations that govern the structure of our DDM ensemble: $\Omega \sim m^{\gamma}$, $\Gamma \sim m^{y}$, and $m_{k} \sim k^{\delta}$. Corresponding to these are three scaling coefficients: $\gamma, y$, and $\delta$. In general, there are many detailed phenomenological constraints that govern the allowed values of these exponents. However, for our purposes in this paper we shall concentrate on only the two most fundamental constraints that ensure the "zerothorder" phenomenological self-consistency of the DDM ensemble as a whole. Indeed, our purpose in this section is not to develop a detailed phenomenological set of bounds on these scaling exponents so much as to understand the general architecture of how these constraints play against each other across the DDM ensemble. A more detailed study of the phenomenological constraints on these scaling relations can be found in Ref. [33].

Our first constraint concerns the decay widths, or equivalently the lifetimes, of our ensemble states. In general, any dark-matter particle which decays too rapidly into SM states is likely to upset big-bang nucleosynthesis (BBN) and light-element abundances, and also leave undesirable imprints in the cosmic microwave background (CMB) and diffuse photon backgrounds. However, if such a decaying particle carries a sufficiently small cosmological abundance at the time of its decay, the disruptive effects of this decay will be minimal and all constraints from BBN, the CMB, etc., can potentially be satisfied. This then leads to the fundamental notion [1] which serves as the underpinning of the DDM framework, namely that dark-matter stability is no longer required in a multicomponent context so long as it is replaced by a balancing of lifetimes against abundances across the dark-matter ensemble, so that states carrying larger abundances are longer-lived while states that are shorter-lived carry smaller abundances. This in turn requires that abundances scale inversely with decay widths, i.e., that

$$
\Omega_{i} \sim \Gamma_{i}^{\alpha} \quad \text { where } \alpha<0 .
$$

With $\Omega \sim m^{\gamma}$ and $\Gamma \sim m^{y}$ we find that $\alpha=\gamma / y$, whereupon Eq. (4.5) yields

$$
\gamma / y<0
$$

Thus $\gamma<0$ corresponds to $y>0$, which for a two-body decay into light fermions leads to the constraint $d \geq 4$, as discussed above.

Strictly speaking, the constraint in Eq. (4.6) should be understood as applying to only those portions of the DDM ensemble consisting of dark-matter components whose decays into SM states have the potential to be phenomenologically problematic. For example, extremely heavy dark-matter states decaying during extremely early periods of cosmological evolution well before $\mathrm{BBN}$ need not satisfy these bounds, as the decay products rapidly thermalize with the radiation bath. Consequently, while such decays can potentially induce a later period of reheating, depending on the abundances of the decaying particles [34], they typically have few other observable consequences. Thus one could conceivably tolerate having $\gamma, y>0$ within such portions of the ensemble. This issue will be discussed in detail in Ref. [33]. Likewise, the cosmological abundances of dark-matter states whose lifetimes significantly exceed $10^{9} t_{\text {now }}$ are also unconstrained and also may exhibit $\gamma, y>0$. Thus, it is only within that all-important region of the DDM ensemble consisting of states with lifetimes $\tau$ in the range $t_{\mathrm{BBN}} \lesssim$ $\tau \lesssim 10^{9} t_{\text {now }}$ that we must demand $\gamma / y<0$.

It is also important to note that in deriving the result in Eq. (4.6), we have not assumed that $\gamma$ is a constant throughout the relevant portion of the DDM ensemble. Likewise, we have also not assumed that $y$ is a constant over this range. Instead, we simply need to verify that Eq. (4.6) holds throughout the relevant portion of the ensemble. Indeed, both $\gamma$ and $y$ are free to vary so long as the constraint in Eq. (4.6) is satisfied.

Our second constraint on these scaling exponents concerns the time-development of the total dark-matter abundance $\Omega_{\mathrm{tot}} \equiv \sum_{i} \Omega_{i}$, or equivalently the time-development of the corresponding energy density $\rho_{\text {tot }}$, where $\Omega_{\text {tot }}=$ $\rho_{\text {tot }} / \rho_{\text {crit }}$. Here $\rho_{\text {crit }}=2 M_{P}^{2} H^{2}$, where $H$ is the Hubble parameter and where $M_{P} \equiv\left(8 \pi G_{N}\right)^{-1 / 2}$ is the reduced Planck scale. If each constituent within the ensemble were stable, the total energy within the ensemble would remain constant, implying that the energy density $\rho_{\text {tot }}$ would fall as a function of time solely because of the Hubble expansion of the universe, with $d \rho_{\text {tot }} / d t=-3 H \rho_{\text {tot }}$. In other words, 
the collective equation-of-state parameter $w_{\text {eff }}$ for the darkmatter ensemble as a whole, defined as [1]

$$
w_{\mathrm{eff}}(t) \equiv-\left(\frac{1}{3 H \rho_{\mathrm{tot}}} \frac{d \rho_{\mathrm{tot}}}{d t}+1\right)
$$

would vanish. The vanishing of $w_{\text {eff }}(t)$ under such stability assumptions is of course consistent with the interpretation of the corresponding energy density of our ensemble as being associated with dark matter (as opposed to dark energy or dark radiation), which is in turn consistent with observational constraints. However, the constituents within our dark ensemble are not stable: as described above, they decay with lifetimes that obey certain scaling relations relative to their cosmological abundances. These decays in turn cause $\Omega_{\text {tot }}$ to fall as a function of time, leading to a positive value of $w_{\text {eff }}$. The scaling relations that govern these decays must therefore be balanced in such a way [1] that $w_{\text {eff }}$ not be too far from zero at the present time and also not have varied significantly within the recent cosmological past. The first of these requirements ensures that we can continue to interpret the energy within the DDM ensemble as associated with dark matter, within experimental constraints. By contrast, the second requirement stems from the observation that the behavior of $w_{\text {eff }}(t)$ has an impact on the expansion history of the universe, independent of the constraint in Eq. (4.6). Indeed, the behavior of $w_{\text {eff }}(t)$ is constrained $[35,36]$ by a combination of CMB data $[19,37]$; observations of baryon acoustic oscillations in galaxies [38-40] and in the Lyman- $\alpha$ forest [41,42]; and measurements of the redshifts and luminosity distances of Type-Ia supernovae [43]. Moreover, modifications to the expansion rate of the universe can also affect the light-element abundances generated during the BBN epoch. Thus, for all practical purposes it is reasonable to identify the "recent past" over which $w_{\text {eff }}(t)$ should not vary significantly as the period since BBN.

In this connection, we note that an equation of state is a property intrinsic to the ensemble. As such, $w_{\text {eff }}(t)$ is independent of the background cosmological epoch. Thus the constraints regarding the behavior of $w_{\text {eff }}(t)$ that we have indicated above are applicable regardless of whether we are considering a matter- or radiationdominated epoch, and likewise also apply across transitions between epochs.

These constraints were investigated in Ref. [1]. It turns out that the constraint that $w_{*} \equiv w_{\text {eff }}\left(t_{\text {now }}\right)$ not be too far from zero is ultimately independent of the scaling exponents and only requires suitable overall normalizations for our abundance and lifetime scaling relations [1]. In other words, the value of $w_{*}$ ultimately depends on the overall prefactor coefficients that come into these scaling relations but not on the scaling exponents themselves. We shall therefore henceforth assume that this constraint has been satisfied and that $w_{*}$, though positive, is extremely small and ultimately within the experimental bounds consistent with an interpretation in terms of dark matter.

By contrast, requiring that $w_{\text {eff }}(t)$ not have varied significantly within the recent past leads directly to a constraint on our scaling exponents [1]. Following the discussion in Ref. [1], this constraint may be phrased as follows. In the limit that our ensemble consists of a large number of densely packed dark-matter states, we can imagine that the spectrum of discrete decay widths $\Gamma_{i}$ is nearly continuous, parametrized by a continuous variable $\Gamma$. In this approximation, we can view the spectrum of abundances $\Omega_{i}$ as a continuous function $\Omega(\Gamma)$ of decay widths. We can likewise express our density of states as a density of states per unit $\Gamma$, henceforth denoted $n_{\Gamma}$. In general, both of these quantities will have scaling behaviors of the form

$$
\Omega(\Gamma) \sim \Gamma^{\alpha}, \quad n_{\Gamma} \sim \Gamma^{\beta},
$$

where $\alpha$ is the same exponent we have already seen in Eq. (4.5). It then turns out [1] that the corresponding equation-of-state parameter $w_{\text {eff }}(t)$ depends only on $w_{*}$ and on the sum $x \equiv \alpha+\beta$. Indeed, $w_{\text {eff }}$ is generally a rather nontrivial function of these variables. However, for $w_{*} \ll 1$, one finds [1]

$$
w_{\text {eff }}(t) \approx w_{*}\left(\frac{t}{t_{\text {now }}}\right)^{-x-1}
$$

Given that $w_{*} \ll 1$, we thus see that we must have $x \leq-1$ in order for $w_{\text {eff }}(t)$ to have remained small throughout the recent past. Again we stress that this conclusion holds even though our definition of "recent past" stretches across both radiation- and matter-dominated epochs.

Of course, strictly speaking, any value of $x \leq-1$ is permitted for those ensemble states decaying within this time interval, even values of $x$ which are significantly less than -1 . Taking $x \ll-1$ simply means that even though $w_{\text {eff }}(t)$ has a very small value $w_{*} \ll 1$ at the present epoch, it approaches zero extremely rapidly as we go backwards in time towards BBN. However, while a choice $x \ll-1$ succeeds in guaranteeing $w_{\text {eff }}(t) \ll 1$ during the entire recent period since BBN, such a choice is unnatural from several points of view. First, if $x \ll 1$ for all ensemble states decaying between BBN and the present epoch, it is natural to assume that similar values of $x$ would continue to hold for states immediately beyond this range, i.e., for states whose decays will occur in the immediate future. However, this would then cause $w_{\text {eff }}(t)$ to experience a sudden dramatic growth for $t>t_{\text {now }}$. While this cannot be ruled out solely on the grounds of equations of state and their behaviors, such a sudden dramatic change in $w_{\text {eff }}(t)$ beyond $w_{\text {eff }} \ll 1$ would effectively single out the present time as a special epoch in the cosmological timeline. Indeed, the only way to avoid this would be to assume that $x$ itself must 
experience a sudden change at the beginning of that portion of the ensemble whose states decay in the present epoch. However, this too would single out the present epoch as special. There may also be more direct phenomenological reasons to exclude having $x \ll-1$. Since nonzero values of $w_{\text {eff }}(t)$ are ultimately due to the decays of our dark-matter constituents, a sudden, rapid growth in $w_{\text {eff }}(t)$ is likely to be correlated with a large injection of decay products, potentially including large amounts of radiation. If these decay products include SM particles, this in turn is likely to cause issues with diffuse photon backgrounds, etc. This too will be discussed in more detail in Ref. [33].

Thus, for such reasons, it is more natural to assume that $x \lesssim-1$. In other words, we shall henceforth assume that $x$, though less than -1 , is not too far below -1 . We shall demand that this be true over that portion of the ensemble whose states decay between BBN and the present epoch. This assumption allows us to avoid sudden changes in either the cosmological evolution or the structure of the ensemble once the current epoch is reached.

It is straightforward to express the constraint $x \lesssim-1$ in terms of our scaling coefficients $\{\gamma, y, \delta\}$. We recall that $x \equiv \alpha+\beta$, and we have already seen below Eq. (4.5) that $\alpha=\gamma / y$. To calculate $\beta$, we observe that

$n_{\Gamma}=n_{m}\left|\frac{d m}{d \Gamma}\right| \sim m^{1 / \delta-1} m^{1-y} \sim \Gamma^{(1 / \delta-1) / y} \Gamma^{(1-y) / y}$,

allowing us to identify $\beta=1 /(y \delta)-1$. We thus find that

$$
x=\frac{\gamma}{y}+\frac{1}{y \delta}-1,
$$

whereupon the constraint $x \lesssim-1$ reduces to

$$
\frac{1}{y}\left(\gamma+\frac{1}{\delta}\right) \lesssim 0
$$

For positive $y$ we thus find

$$
y>0: \quad \gamma \lesssim-1 / \delta,
$$

while for negative $y$ we find

$$
y<0: \quad \gamma \gtrsim-1 / \delta .
$$

In either case, these inequalities can be rewritten in the common form

$$
\delta \gtrsim \delta_{\min } \equiv-1 / \gamma
$$

Indeed, this constraint holds regardless of the sign of $y$.

Remarkably, we see that the scaling exponent $y$ has completely dropped out of this constraint. Moreover, we observe that this constraint algebraically has the same form as the constraint that would emerge from demanding that $\Omega_{\text {tot }}$ be finite in cases where our ensemble consists of an infinite tower of states whose masses stretch to infinity. In such cases we would have

$$
\Omega_{\mathrm{tot}}=\int d m n_{m} \Omega(m) \sim \int d m m^{1 / \delta-1} m^{\gamma},
$$

and we see that the "ultraviolet" finiteness of this integral requires that $\gamma \leq-1 / \delta$. Of course, despite their algebraic similarity, at a physical level these are ultimately different constraints since the constraint stemming from Eq. (4.16) applies for either sign of $y$ and applies only for those states at the large-mass "ultraviolet" end of the ensemble, while the constraint in Eq. (4.13) assumes that $y$ is positive and needs only apply within that portion of the ensemble whose states decay between BBN and the present epoch. It is nevertheless interesting that both constraints, operating over different portions of the ensemble, share a common algebraic structure.

Just as with the constraint in Eq. (4.6), we emphasize that Eq. (4.15) must hold only throughout the relevant portions of the ensemble discussed above. Indeed, both $\gamma$ and $\delta$ are free to vary so long as Eq. (4.15) holds at each mass scale within this region.

As indicated above, the constraint in Eq. (4.15) can be satisfied with $\gamma$ either positive or negative. Indeed, in either case we need only require that $y$ and $\gamma$ have opposite signs, in accordance with Eq. (4.6). However, these results help to explain why the situation with $\gamma<0$ is more natural from a DDM perspective. Note that $\delta$ is necessarily positive, since our states are ordered in terms of increasing mass by construction. Moreover, when $\gamma$ is negative, we find that $\delta_{\min }$ is positive. This implies that $\delta$ can indeed easily satisfy $\delta \gtrsim \delta_{\min }$-i.e., it is not difficult for $\delta$ to be only slightly greater than $\delta_{\min }$. For $\gamma<0$, by contrast, $\delta_{\min }$ is negative. Thus, although any positive value of $\delta$ is greater than $\delta_{\min }$, it can be difficult for $\delta$ to be both positive and only slightly greater than $\delta_{\min }$. Indeed, this latter requirement becomes increasingly hard to satisfy when $\gamma$ is small. There are also other reasons to prefer $\gamma<0$. In general, it is more natural for heavier states to have smaller lifetimes and larger decay widths than lighter states. This requires $y>0$, which in turn requires $\gamma<0$. Indeed, it is for all of these reasons that it has been crucial to find ways in which we might obtain abundances with $\gamma<0$ from thermal freeze-out. Of course, within other regions of the ensemble, no such constraints need apply. Such regions could then have $\gamma>0$, even while $\delta, y>0$.

Turning back to Eq. (4.15), we see that for each value of $\gamma$ there is a corresponding minimum mass-distribution scaling exponent $\delta_{\min }$ that is permissible in order to ensure that $x \lesssim-1$. In Fig. 6, we have plotted $\delta_{\min }$ as a function of $m$ for different values of $m_{\phi} / m_{\psi}$. In each case we have plotted results for $\Delta \gamma=0,2,4$, corresponding to the curves 

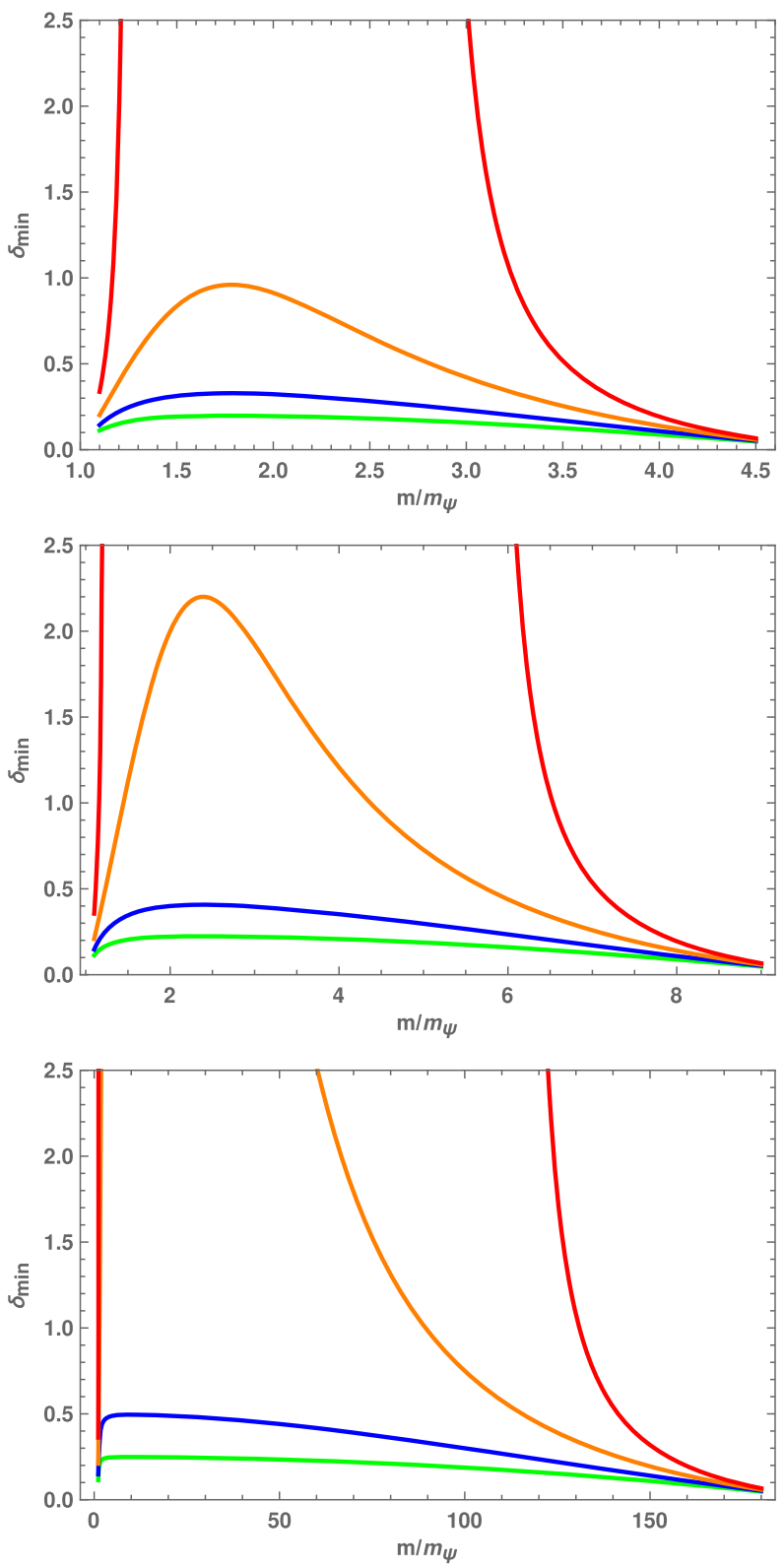

FIG. 6. Minimum $\delta$-exponents $\delta_{\min } \equiv-1 / \gamma$, plotted as functions of $\mathrm{m} / \mathrm{m}_{\psi}$ for $m_{\phi} / m_{\psi}=10$ (top panel), 20 (middle panel), and 400 (bottom panel). Each panel shows results for $\Delta \gamma=-2$ (green), 0 (blue), 2 (orange), and 4 (red); for $\Delta \gamma \geq 0$ these results correspond to the $\Omega$ plots shown in Figs. 4 and 5. For $\Delta \gamma<2$, we see that $\delta_{\min }$ remains finite for all $m_{\phi} / m_{\psi}$. This remains true even in the $\Delta \gamma=2$ case, for which there exist certain mass regions in which $\delta_{\text {min }}$ grows significantly as $m_{\phi} / m_{\psi} \rightarrow \infty$, reflecting the increasing tendency of the corresponding abundances $\Omega$ to become almost flat as functions of $m$. For $\Delta \gamma=4$, by contrast, $\delta_{\text {min }}$ actually diverges at the two masses for which the corresponding values of $\Omega$ in Figs. 4 and 5 reach local minima or maxima. Between these masses $\gamma>0$ and the constraint in Eq. (4.15) becomes vacuous.

in Figs. 4 and 5, and we have also shown results for $\Delta \gamma=-2$, corresponding to the case in which thermal freeze-out occurs due to dark-matter annihilation into pairs of spin-1 particles. We immediately see that for the entire allowed mass range, the cases with $\Delta \gamma=-2$ and $\Delta \gamma=0$ are consistent with not only $\delta=1$, corresponding to a DDM ensemble consisting of the KK states resulting from circle compactification, but also $\delta=1 / 2$, corresponding to a DDM ensemble of stringlike origin. However, we also learn that these constraints become more exclusive as $\Delta \gamma$ increases, and tend to allow such values of $\delta$ only within certain mass regions. For example, we see that an ensemble of KK states is consistent with the abundances produced through a thermal production process with $\Delta \gamma=2$ and $m_{\phi} / m_{\psi} \gg 1$ only within the approximate mass range $\mathrm{m} / \mathrm{m}_{\psi} \gtrsim 100$. Finally, we note that $\delta_{\min }$ is negative within those mass ranges for which $\gamma>0$ in Figs. 4 and 5. The constraint in Eq. (4.15) is then vacuous, and no value of $\delta_{\min }$ is plotted in Fig. 6 .

Depending on which portions of the allowed mass ranges are actually populated by the constituents of our DDM ensemble, we see that entirely different dark-matter phenomenologies can emerge. As a result of the constraints in Eqs. (4.6) and (4.15), we see that the different classes of phenomenologically allowed thermal freeze-out processes, as well as different classes of dark-matter decay processes, are closely tied to the structure of (and indeed the corresponding distribution of masses across) the DDM ensemble. This then provides important correlations between the particle physics of the ensemble structure, the particle physics of the annihilations of its constituents, and the overall (in this case, thermal) cosmological history in which this particle physics is embedded.

\section{DISCUSSION AND CONCLUSIONS}

Within the DDM framework, the phenomenological viability of the dark-matter ensemble is the result of the interplay between three fundamental relations which govern how the masses, cosmological abundances, and decay widths of the individual ensemble constituents scale relative to one another across the ensemble as a whole. While the scaling relations for masses and decay widths primarily depend on particle-physics considerations alone, the scaling relation for cosmological abundances typically depends on a mix of particle-physics and cosmological considerations. Thus, any concrete realization of the DDM framework relies on there being an appropriate abundance-generation mechanism which not only arises naturally within the corresponding cosmological model but also gives rise to an abundance function $\Omega(m)$ with a suitable scaling behavior.

Most prior work realizing explicit DDM ensembles has relied on nonthermal abundance-production mechanisms such as vacuum misalignment, as these can easily give rise to scaling relations in which the abundances scale inversely with mass. As we have discussed, phenomenological constraints tend to prefer this behavior over large portions of any DDM ensemble. Unfortunately, the simplest thermal freeze-out mechanisms tend to result in abundances with 
the opposite behavior, growing as a function of mass. Indeed, this behavior is an intrinsic element underpinning the so-called "WIMP miracle." The purpose of this paper has therefore been to determine whether the scaling behavior desired for DDM ensembles might also be realized through thermal freeze-out. This would in turn determine whether the DDM framework can be extended into the thermal domain.

The results of this paper provide an answer in the affirmative. Indeed, we have shown that relatively straightforward modifications to standard dark-matter annihilation processes result in freeze-out abundances which fall, rather than rise, as functions of constituent mass. In fact, we have found that a whole spectrum of behaviors is possible, with abundances that can fall within certain portions of our DDM ensemble and rise within others. Indeed, by adjusting the values of the discrete dark-sector mass spectrum $\left\{m_{i}\right\}$, we can even continuously dial our abundance scaling exponents $\gamma$ across a wide range of values. The results of this paper thus suggest that a rich and flexible darkmatter phenomenology can result when the DDM framework is extended into the thermal domain. This in turn can provide a versatile tool for model-building within the DDM framework. Indeed, we note that the typical mass window for successful thermal freeze-out is $\mathcal{O}(1) \mathrm{keV} \lesssim m \lesssim$ $100 \mathrm{TeV}$. Since this is also the range of mass scales for which direct-detection experiments and collider searches for missing transverse energy are typically sensitive, the kinds of DDM ensembles whose constituents receive their abundances from thermal freeze-out are also the kinds of ensembles capable of giving rise to the wealth of distinctive phenomenological signatures discussed in Refs. [3-5]signatures which can potentially serve to distinguish these ensembles from traditional dark-matter candidates. Thus we expect thermal DDM ensembles to have immediate implications for these kinds of experimental signatures and bounds-even potentially more than for their nonthermal cousins.

In general, a collection of dark-matter states will sequentially undergo freeze-out in order of their masses $m_{i}$, with the heaviest states freezing out first. Indeed, freeze-out of a given particle generally occurs when the temperature $T$ of the universe is approximately the mass of the particle, with $x_{i} \equiv m_{i} / T \approx \mathcal{O}(20)$. However, there are subleading logarithmic effects which allow certain states to freeze out with slightly smaller values of $x_{i}$ than others, and these subleading logarithmic effects depend on the cross-sections $\left\langle\sigma_{i} v\right\rangle$. It is these effects which ultimately determine the cosmological abundances with which the states emerge after freeze-out. Thus, while the masses $m_{i}$ of the states in a given ensemble determine the order of freeze-out as a function of time, it is the corresponding cross-sections $\left\langle\sigma_{i} v\right\rangle$ which determine the order of freeze-out as a function of $x$. These are, of course, general statements concerning the physics of the freeze-out process, and they remain true in our scenarios as well. However, what we have shown in this paper is that while we cannot adjust the order in which our ensemble constituents freeze out as a function of time, we can certainly adjust the order in which they freeze out as a function of $x$. Indeed, what we have shown is that there exist annihilation processes for which the cross-sections $\left\langle\sigma_{i} v\right\rangle$ induce the lighter states to freeze out with smaller values of $x$, thereby imparting larger abundances to these states and producing the desired negative scaling exponent $\gamma<0$. Moreover, as we have seen, it is even possible to arrange our states to freeze out in a nonmonotonic order as a function of $x$, with the states sitting at the local maxima of our abundance curves in Figs. 4 and 5 freezing out at the smallest values of $x$ and those at local minima freezing out at the largest.

We are not the first to demonstrate that thermal freezeout can yield abundance scaling relations that differ from those associated with the traditional WIMP paradigm. Recall that standard thermal freeze-out during a radiationdominated (RD) epoch yields a dark-matter abundance which scales with the freeze-out temperature $T_{f}$, the mass $m_{\chi}$ of the dark-matter particle, and the thermally averaged annihilation cross-section $\langle\sigma v\rangle$ according to the relation

$$
\Omega_{\chi} \propto \frac{m_{\chi}}{T_{f}\langle\sigma v\rangle} .
$$

The ratio $m_{\chi} / T_{f}$ is famously independent of $\langle\sigma v\rangle$ up to logarithmic corrections. Thus, in traditional WIMP scenarios, in which $\langle\sigma v\rangle \propto g_{\chi}^{4} / m_{\chi}^{2}$, one recovers Eq. (1.2). However, in scenarios with nonstandard cosmological histories in which dark-matter freeze-out does not take place during a RD epoch, this scaling relation is altered. One example is the case in which freeze-out occurs immediately prior to a late period of reheating-i.e., during an epoch in which the universe is dominated by a nonrelativistic particle species (or by the zero mode of a rapidly oscillating scalar field) which is continually decaying into radiation. In such scenarios, provided that $\langle\sigma v\rangle$ is sufficiently large that thermal equilibrium is established between the dark-matter particles and the radiation bath, one finds $[44,45]$

$$
\Omega_{\chi} \propto \frac{m_{\chi} T_{\mathrm{RH}}^{3}}{T_{f}^{4}\langle\sigma v\rangle}
$$

where $T_{\mathrm{RH}}$ is the reheat temperature associated with this late period of reheating. By contrast, if $\langle\sigma v\rangle$ is small and the dark matter never thermalizes, the dark matter "freezes in" [46] rather than freezing out, and one finds [45]

$$
\Omega_{\chi} \propto \frac{T_{\mathrm{RH}}^{7}\langle\sigma v\rangle}{m_{\chi}^{5}} .
$$

Likewise, if thermal freeze-out occurs during an epoch in which the energy density of the universe is dominated 
by the kinetic energy associated with a rapidly rolling scalar-field zero mode (as in so-called "kination" scenarios [47-49]), one finds [50]

$$
\Omega_{\chi} \propto \frac{m_{\chi}}{T_{\mathrm{RH}}\langle\sigma v\rangle},
$$

up to logarithmic corrections in $T_{f} / T_{\mathrm{RH}}$. All of these scenarios alter the standard $m_{\chi}$-dependence for the resulting cosmological abundance.

There are also a variety of nonthermal mechanisms through which a sizable dark-matter abundance can be generated. As one might expect, these mechanisms lead to altogether different scaling relations for $\Omega_{\chi}$ as a function of $m_{\chi}$. One such mechanism is production through the decays of some other, heavier particle which comes to dominate the energy density of the universe at early times. In the regime in which the dark-matter particles produced in this way are effectively decoupled from the radiation, the contribution to $\Omega_{\chi}$ is proportional [51] to the fraction $f_{\chi}$ of the energy of the heavy decaying particle that is transferred to $\chi$ (rather than to other decay products) and is approximately independent of $m_{\chi}$. By contrast, in the opposite regime in which $\langle\sigma v\rangle$ is sufficiently large that the dark-matter particles produced by such decays undergo significant annihilation, the number density $n_{\chi}$ of such particles is depleted by annihilation to $n_{\chi} \sim H\left(T_{\mathrm{RH}}\right) /\langle\sigma v\rangle$, where $H\left(T_{\mathrm{RH}}\right)$ is the value of the Hubble parameter at the reheat temperature associated with the late period of reheating induced by the decay of the heavy particle. In this case, one finds [52]

$$
\Omega_{\chi} \propto \frac{m_{\chi}}{T_{\mathrm{RH}}\langle\sigma v\rangle} .
$$

Of course, other nonthermal mechanisms for abundance generation exist as well. These include, for example, misalignment production as well as production via the decays of topological defects (cosmic strings, domain walls, etc.) or nontopological solitons (e.g., oscillons [53-55]). These abundance-generation mechanisms each have their own characteristic scaling relations with $m_{\chi}$ and with the other relevant parameters of the theory.

These examples illustrate the ways in which modified cosmologies can produce a variety of possible scaling relations between the cosmological abundance $\Omega_{\chi}$ and quantities such as $m_{\chi}$ and $\langle\sigma v\rangle$. Indeed, by invoking an early period of matter domination, kination, etc., one can achieve almost any scaling behavior one desires. However, we have shown in this paper that such departures from the standard cosmology are not necessary in order to obtain the appropriate scaling relations for a DDM ensemble through thermal freeze-out. Indeed, we have shown that the desired relations arise naturally within the standard cosmology and are realized in a particularly simple class of particle-physics models. In other words, our approach to modifying the traditional scaling relations expected from thermal freezeout has involved modifying the particle physics rather than modifying the cosmological narrative in which the particle physics is embedded.

Our results in this paper suggest many areas for further research. First, it would be interesting to explore the phenomenology that might result from thermal freezeout due to other, more complex annihilation processes. For example, given that the DDM framework involves large multiplicities of dark-matter states, it might be interesting to consider a strongly interacting massive particle (SIMP) framework $[56,57]$ within which dark-matter annihilation might receive significant contributions from $3 \rightarrow 2$ and perhaps even $4 \rightarrow 2$ processes in which dark-sector particles annihilate into other dark-sector particles. Likewise, in this paper we have assumed that our dominant annihilation mode is one in which our dark-sector ensemble components $\chi_{i}$ annihilate to states $\psi$ which are outside the ensemble. However, in a multicomponent framework such as the DDM framework, there is also the possibility of intra-ensemble annihilation processes of the form $\bar{\chi}_{i} \chi_{i} \rightarrow$ $\bar{\chi}_{j} \chi_{j}$ where $\chi_{j}$ is lighter than $\chi_{i}$, as well as coannihilation processes of the form $\bar{\chi}_{i} \chi_{j} \rightarrow \bar{\psi} \psi$ with $i \neq j$. Such processes can potentially alter the freeze-out process in nontrivial ways. Moreover, the existence of such processes also implies the existence of inelastic scattering processes of the form $\chi_{i} \psi \rightarrow \chi_{j} \psi$, which can potentially also impact the dynamics of freeze-out.

Second, throughout our analysis we have assumed that our dark-matter/mediator couplings $g_{\chi}$ are universal throughout the ensemble. It might therefore be of interest to allow these couplings to vary across the DDM ensemble, thereby introducing an additional mass-dependence into the resulting cosmological abundances. In general, the constituent-dependent dark-matter/mediator coupling $g_{i}$ is the coupling associated with each constituent at its own freezeout temperature. Such couplings $g_{i}$ will therefore experience an automatic effective "running" as we successively integrate out lighter and lighter states from the ensemble. Indeed, such running has already been calculated for the case in which the ensemble is a tower of KK modes [58].

Third, in this paper, we have restricted our analysis to situations in which all of the DDM constituents freeze out while nonrelativistic. It is nevertheless also possible that very light, very feebly coupled ensemble constituents could potentially freeze out while still relativistic. This could then significantly modify the resulting scaling behaviors in phenomenologically important ways.

Fourth, in this paper we have only examined the most immediate, "zeroth-order" constraints that might affect our overall scaling relations. There are, of course, many other more detailed constraints that must be imposed before building a viable DDM model. These are ultimately constraints coming from potential signatures of thermal DDM scenarios within direct-detection, indirect-detection, 
and collider experiments. The case of indirect detection is particularly interesting, as there is the possibility of a correlation - and even a complementarity-between the fluxes of end-products from dark-matter annihilation and dark-matter decay. While viable DDM models have been constructed $[2,11]$ which satisfy all known experimental and observational constraints on the dark-matter sector, these models relied on nonthermal abundance-production mechanisms. It still remains to determine the detailed phenomenological constraints that must be imposed within a thermal context, and then to build actual models of this type. Indeed, this paper represents only the first step in this direction.

Finally, it would be interesting to examine in more detail the model-building possibilities that emerge from having abundances which fall as a function of mass within one part of a DDM ensemble and rise within another, thereby experiencing different values of the scaling exponent $\gamma$ within different regions of the ensemble. Given that the different portions of the DDM ensemble can be relevant at different cosmological time scales due to the variations in their masses and lifetimes, this flexibility may enable a single DDM ensemble to simultaneously address many thorny phenomenological challenges that at first glance might otherwise appear to be disconnected or perhaps even contradictory.

We conclude this paper with an important comment. Our aim throughout this paper has been to examine the scaling relations which govern a thermal DDM ensemble with as much generality as possible, without reference to specific mass or energy scales. From a phenomenological perspective, however, given the numerous observational constraints and consistency conditions that apply to thermal freeze-out scenarios, it is important to assess the natural values for the masses and couplings which characterize such an ensemble.

In order to obtain a sense of the physical scales involved, we begin by recalling that both in canonical WIMP scenarios and in the $m_{i} \gg m_{\phi}, m_{\psi}$ regime of our DDM analysis, $\Omega_{i}$ is essentially determined by the ratio $g_{\chi}^{2} g_{\psi}^{2} / m_{i}^{2}$. The WIMP miracle is essentially the observation that an abundance $\Omega_{i} \approx \Omega_{\mathrm{CDM}}$ is obtained for $m_{i} \approx 250 \mathrm{GeV}$ and $g_{\psi}=g_{\chi} \approx 0.65$, which yields

$$
\frac{m_{i}^{2}}{g_{\chi}^{2} g_{\psi}^{2}} \sim 0.35 \mathrm{TeV}^{2}
$$

By contrast, in the $m_{\phi} \gg m_{i}, m_{\psi}$ regime of our DDM model, the corresponding quantity which determines the cross-section is $16 g_{\chi}^{2} g_{\psi}^{2} m_{i}^{2} / m_{\phi}^{4}$. For an ensemble of particles in this regime, Eq. (2.7) implies that $\Omega_{\text {tot }} \propto \sum_{i}\left\langle\sigma_{i} v\right\rangle^{-1}$. It therefore follows that in order to reproduce the observed value of $\Omega_{\mathrm{CDM}}$, such an ensemble must satisfy

$$
\sum_{i} \frac{m_{\phi}^{4}}{16 g_{\chi}^{2} g_{\psi}^{2} m_{i}^{2}} \lesssim 0.35 \mathrm{TeV}^{2}
$$

However, by the same token, a total abundance $\Omega_{\text {tot }}>$ $\Omega_{\mathrm{CDM}}$ is problematic, signifying overproduction of dark matter. This consideration then implies the constraint

$$
\sum_{i}\left(\frac{m_{\phi}}{m_{i}}\right)^{2} \lesssim g_{\chi}^{2} g_{\psi}^{2}\left(\frac{2.37 \mathrm{TeV}}{m_{\phi}}\right)^{2} .
$$

The masses $m_{i}$ and the parameters $g_{\chi}, g_{\psi}$, and $m_{\phi}$ are constrained by other considerations as well. On the one hand, we have assumed that $m_{\phi} \gg m_{i}$ for all $i$. On the other hand, perturbativity considerations require that $g_{\chi}^{2}, g_{\psi}^{2} \lesssim 4 \pi$.

Imposing all of these constraints, we find that the preferred regime for a thermal DM ensemble is one in which $g_{\chi}$ and $g_{\psi}$ are large and in which $m_{i} \ll m_{\phi} \ll$ $\mathcal{O}(\mathrm{TeV})$. However, this is easy to arrange, for example, in scenarios in which the dark and visible sectors are approximately decoupled and the ensemble constituents annihilate primarily into other, lighter dark-sector states. Indeed, such scenarios can accommodate cold relic particle masses as low as $\mathcal{O}(\mathrm{keV})$ [21]. It is worth noting that hidden-sector dark-matter models of this sort have a rich phenomenology despite their suppressed couplings between the dark and visible sectors (for a review, see, e.g., Ref. [59]). We also note that we can always raise the mass scale of the mediator and the dark matter simply by increasing the annihilation cross-section. This can be done, for example, by adding more final states $\psi$ into the annihilation process, as might be arranged if $\psi$ were to carry something analogous to a color quantum number. We also note that in the context of modified cosmologies-for example, in scenarios in which $\Omega_{\text {tot }}$ is diluted by entropy injection after the freeze-out of the lightest constituent- the bound in Eq. (5.8) can be considerably weakened. Thus, in such scenarios, an even broader range of mass scales for thermal DDM ensembles becomes accessible.

\section{ACKNOWLEDGMENTS}

We are happy to thank Kimberly Boddy and Jeff Kost for useful discussions. We are also particularly grateful for extensive conversations with David Curtin and Adrienne Erickcek which helped to influence various aspects of this paper. K. R. D., J. K., and B. T. would also like to thank the Center for Theoretical Underground Physics and Related Areas (CETUP*) for its hospitality and partial support during the 2016 Summer Program; likewise, K. R. D. and B. T. would also like to acknowledge the hospitality of the Aspen Center for Physics, which is supported in part through NSF Grant No. PHY-1607611. The research activities of K.R.D. are supported in part by the Department of Energy under Grant No. DE-FG0213ER41976 (DE-SC0009913) and by the National Science Foundation through its employee IR/D program. The research activities of J. K. are supported in part by NSF CAREER Grant No. PHY-1250573, and those of B. T. are supported in part by NSF Grant No. PHY-1720430. The opinions and conclusions expressed herein are those of the authors, and do not represent any funding agencies. 
[1] K. R. Dienes and B. Thomas, Phys. Rev. D 85, 083523 (2012).

[2] K. R. Dienes and B. Thomas, Phys. Rev. D 85, 083524 (2012).

[3] K. R. Dienes, S. Su, and B. Thomas, Phys. Rev. D 86, 054008 (2012).

[4] K. R. Dienes, S. Su, and B. Thomas, Phys. Rev. D 91, 054002 (2015).

[5] K. R. Dienes, J. Kumar, and B. Thomas, Phys. Rev. D 86, 055016 (2012).

[6] K. R. Dienes, J. Kumar, and B. Thomas, Phys. Rev. D 88, 103509 (2013).

[7] K. K. Boddy, K. R. Dienes, D. Kim, J. Kumar, J. C. Park, and B. Thomas, Phys. Rev. D 94, 095027 (2016).

[8] K. K. Boddy, K. R. Dienes, D. Kim, J. Kumar, J. C. Park, and B. Thomas, Phys. Rev. D 95, 055024 (2017).

[9] K. R. Dienes, J. Kumar, B. Thomas, and D. Yaylali, Phys. Rev. Lett. 114, 051301 (2015).

[10] K. R. Dienes, J. Kumar, B. Thomas, and D. Yaylali, Phys. Rev. D 96, 115009 (2017).

[11] K. R. Dienes and B. Thomas, Phys. Rev. D 86, 055013 (2012).

[12] K. R. Dienes, F. Huang, S. Su, and B. Thomas, Phys. Rev. D 95, 043526 (2017).

[13] K. R. Dienes, J. Fennick, J. Kumar, and B. Thomas, Phys. Rev. D 93, 083506 (2016).

[14] D. Chialva, P. S. B. Dev, and A. Mazumdar, Phys. Rev. D 87, 063522 (2013).

[15] E. W. Kolb and M. S. Turner, Front. Phys. 69, 1 (1990).

[16] P. Gondolo and G. Gelmini, Nucl. Phys. B360, 145 (1991).

[17] G. Jungman, M. Kamionkowski, and K. Griest, Phys. Rep. 267, 195 (1996).

[18] S. Dodelson, Modern Cosmology (Academic Press, Amsterdam, Netherlands, 2003).

[19] P. A. R. Ade et al. (Planck Collaboration), Astron. Astrophys. 594, A13 (2016).

[20] J. L. Feng and J. Kumar, Phys. Rev. Lett. 101, 231301 (2008).

[21] J. L. Feng, H. Tu, and H. B. Yu, J. Cosmol. Astropart. Phys. 10 (2008) 043.

[22] K. Griest and M. Kamionkowski, Phys. Rev. Lett. 64, 615 (1990).

[23] A. Berlin, D. Hooper, and G. Krnjaic, Phys. Lett. B 760, 106 (2016).

[24] Ya. B. Zeldovich, Adv. Astron. Astrophys. 3, 241 (1965).

[25] H. Y. Chiu, Phys. Rev. Lett. 17, 712 (1966).

[26] G. Steigman, Annu. Rev. Nucl. Part. Sci. 29, 313 (1979).

[27] R. J. Scherrer and M. S. Turner, Phys. Rev. D 33, 1585 (1986); 34, 3263(E) (1986).

[28] M. Ibe, H. Murayama, and T. T. Yanagida, Phys. Rev. D 79, 095009 (2009).

[29] W. L. Guo and Y. L. Wu, Phys. Rev. D 79, 055012 (2009).

[30] M. Duch and B. Grzadkowski, J. High Energy Phys. 09 (2017) 159.
[31] J. Kumar and D. Marfatia, Phys. Rev. D 88, 014035 (2013).

[32] M. Srednicki, R. Watkins, and K. A. Olive, Nucl. Phys. B310, 693 (1988).

[33] K. R. Dienes, J. Kumar, P. Stengel, and B. Thomas (unpublished).

[34] J. Fan, O. Özsoy, and S. Watson, Phys. Rev. D 90, 043536 (2014).

[35] G. Blackadder and S. M. Koushiappas, Phys. Rev. D 90, 103527 (2014).

[36] G. Blackadder and S. M. Koushiappas, Phys. Rev. D 93, 023510 (2016).

[37] G. Hinshaw et al. (WMAP Collaboration), Astrophys. J. Suppl. Ser. 208, 19 (2013).

[38] L. Anderson et al. (BOSS Collaboration), Mon. Not. R. Astron. Soc. 441, 24 (2014).

[39] F. Beutler, C. Blake, M. Colless, D. Heath Jones, L. Staveley-Smith, L. Campbell, Q. Parker, W. Saunders, and F. Watson, Mon. Not. R. Astron. Soc. 416, 3017 (2011).

[40] A. J. Ross, L. Samushia, C. Howlett, W. J. Percival, A. Burden, and M. Manera, Mon. Not. R. Astron. Soc. 449, 835 (2015).

[41] T. Delubac et al. (BOSS Collaboration), Astron. Astrophys. 574, A59 (2015).

[42] A. Font-Ribera et al. (BOSS Collaboration), J. Cosmol. Astropart. Phys. 05 (2014) 027.

[43] M. Betoule et al. (SDSS Collaboration), Astron. Astrophys. 568, A22 (2014).

[44] G. F. Giudice, E. W. Kolb, and A. Riotto, Phys. Rev. D 64, 023508 (2001).

[45] A. L. Erickcek, Phys. Rev. D 92, 103505 (2015).

[46] L. J. Hall, K. Jedamzik, J. March-Russell, and S. M. West, J. High Energy Phys. 03 (2010) 080.

[47] B. Spokoiny, Phys. Lett. B 315, 40 (1993).

[48] M. Joyce, Phys. Rev. D 55, 1875 (1997).

[49] P. G. Ferreira and M. Joyce, Phys. Rev. D 58, 023503 (1998).

[50] K. Redmond and A. L. Erickcek, Phys. Rev. D 96, 043511 (2017).

[51] G. B. Gelmini and P. Gondolo, Phys. Rev. D 74, 023510 (2006).

[52] G. Arcadi and P. Ullio, Phys. Rev. D 84, 043520 (2011).

[53] I. L. Bogolyubsky and V. G. Makhankov, Pis'ma Zh. Eksp. Teor. Fiz. 24, 15 (1976).

[54] M. Gleiser, Phys. Rev. D 49, 2978 (1994).

[55] E. J. Copeland, M. Gleiser, and H.-R. Muller, Phys. Rev. D 52, 1920 (1995).

[56] E. D. Carlson, M. E. Machacek, and L. J. Hall, Astrophys. J. 398, 43 (1992).

[57] Y. Hochberg, E. Kuflik, T. Volansky, and J. G. Wacker, Phys. Rev. Lett. 113, 171301 (2014).

[58] S. Bauman and K. R. Dienes, Phys. Rev. D 85, 125011 (2012).

[59] J. L. Feng, Annu. Rev. Astron. Astrophys. 48, 495 (2010). 